\title{
Desarrollo de modelos ecológicos para carbono y nitrógeno en lagunas facultativas secundarias
}

\author{
Developing Ecological Models on Carbon and Nitrogen \\ in Secondary Facultative Ponds
}

\author{
Aponte-Reyes Alexander \\ Grupo de Investigación Saneamiento Ambiental \\ Universidad del Valle, Cali, Colombia \\ Correo: alexander.aponte.reyes@correounivalle.edu.co
}

Información del artículo: recibido: febrero de 2013, reevaluado: abril de 2013, aceptado: julio de 2013

\section{Resumen}

El modelo se formuló para COT y $\mathrm{CO}_{2}, \mathrm{y} \mathrm{NH}_{4}{ }^{+}, \mathrm{NO}_{3}{ }^{-}$y $\mathrm{NTK}$, a partir de información de literatura y lo que se obtuvo en campo durante el seguimiento a tres unidades piloto de laguna facultativa secundaria: laguna convencional, LC, laguna de bafles, LB y laguna de bafles-mallas LBM. Los modelos mostraron sensibilidad a las variables caudal de entrada, radiación solar, $\mathrm{pH}$ y contenido de oxígeno; a nivel de parámetros se encontró que el modelo de Carbono resultó sensible a: $\mathrm{K}_{\text {COT Ba }}, u_{\max B a^{\prime}} u_{\max \mathrm{Al}^{\prime}} \mathrm{K}_{1 \mathrm{OX}}, \mathrm{V}_{\mathrm{A}^{\prime}}, \mathrm{R} \mathrm{D}_{\mathrm{CH}^{\prime}}, \mathrm{YB} \mathrm{B}_{\mathrm{h}}$. El modelo de Nitrógeno resultó sensible a los parámetros: $\mathrm{K}_{\mathrm{COT} \mathrm{Ba}} \mathrm{u}_{\max \text { Ba }} \mathrm{u}_{\max \mathrm{Al}}$ $\mathrm{V}_{\mathrm{A}} \mathrm{K}_{\mathrm{OPH}}, \mathrm{K}_{\mathrm{OPA}}, \mathrm{r}_{4 \mathrm{An}}$. Las pruebas t pareada realizadas indicaron que el modelo de Carbono reprodujo de manera confiable el comportamiento del COT en una laguna facultativa secundaria; mientras que el modelo de Nitrógeno lo hizo para el caso de $\mathrm{NH}_{4}^{+}$. Diferentes topologías afectan la ecología del sistema promoviendo distintas rutas de transformación del nitrógeno; la topología LBM podría alcanzar transformaciones de $C$ empleando volúmenes inferiores, sin embargo se requiere una calibración de los modelos propuestos. Los modelos podrían ser acoplados a modelos hidrodinámicos para un modelado eco-hidrodinámico.

\section{Descriptores:}

- ecología

- modelado

- lagunas facultativas

- biorremediación 


\begin{abstract}
Ecological models formulated for TOC, $\mathrm{CO}_{2}, \mathrm{NH}_{4}^{+}, \mathrm{NO}_{3}^{-}$and NTK, based in literature reviewed and field work were obtained monitoring three facultative secondary stabilization ponds, FSSP, pilots: conventional pond, CP, baffled pond, $B P$, and baffled-meshed pond, BMP. Models were sensitive to flow inlet, solar radiation, $p H$ and oxygen content; the sensitive parameters in Carbon Model were $K_{\text {СОT Ba' }} u_{\max B a^{\prime}} u_{\max A l^{\prime}} K_{1 O X^{\prime}} V_{A l^{\prime}} R 1 D_{C H y^{\prime}} Y B_{h^{\prime}}$. The sensitive parameters in the Nitrogen model were $K_{\text {Сот Ba' }} u_{\max B a^{\prime}} u_{\max A l^{\prime}} V_{A l^{\prime}} K_{O P H^{\prime}} K_{O P A}, r_{4 A n}$. The test $t$-paired showed a good simulating of Carbon model refers to TOC in FSSP; on the other side, the Nitrogen model showed a good simulating of $\mathrm{NH}_{4}^{+}$. Different topological models modify ecosystem ecology forcing different transformation pathways of $\mathrm{Ni}$ trogen; equal transformations of the Carbon BMP topology could be achieved using lower volumes, however, a calibration for a new model would be required. Carbon and Nitrogen models developed could be coupled to hydrodynamics models for better modeling of FSSP.
\end{abstract}

\section{Introducción}

Controlar la contaminación hídrica ocasionada por aguas residuales municipales tiene justificación en problemas ambientales como la generación de gases efecto invernadero, GEI, la presencia de disruptores endocrinos (Janex et al., 2009), la eutrofización de fuentes de agua (Zimmo et al., 2004; Wallace y Austin, 2008) y el deterioro de la calidad de las fuentes de agua. Un enfoque ecológico en el estudio de lagunas facultativas secundarias, LFS (figura 1), apunta a conocer las relaciones establecidas entre la estructura de este ecosistema y las funciones que cumple. Un proceso de simulación-modelación puede explicar dichas relaciones, haciéndolo una herramienta útil para su análisis (Jamu y Piedrahita, 2002). Para analizar una LFS es posible apelar a procedimientos matemáticos que analicen los fenómenos multidimensionales (Legendre y Legendre, 2004) que suceden en estos complejos ecosistemas; el modelado puede integrar variables hidrodinámicas y cinéticas y es una herramienta con amplias posibilidades en el campo del tratamiento de las aguas residuales (Zima et al., 2009; Alvarado et al., 2012; Sah, 2009; Beran y Kargi, 2005; Houweling et al., 2008; Abbas et al., 2006). Disponer de modelos de LFS que conjuguen su ecología, incorporen sus propiedades dinámicas, componentes y variables (Jamu y Piedrahita, 2002) posibilitará la búsqueda de un ambiente sano con criterios de justicia ambiental (Rees, 2008).

La abundancia y disponibilidad del carbono, C, y nitrógeno, $\mathrm{N}$, en la biósfera como constituyentes de la materia orgánica, dependen de transformaciones biogeoquímicas (Jenkins y Zehr, 2008) como las existentes en un LFS, además regulan la productividad de los eco- sistemas acuáticos y terrestres, pues posibilitan la existencia de los productores primarios (Lampert y Sommer, 2007). Por otro lado, el modelado de la generación de gases efecto invernadero desde LFS podría validar modelos ecológicos que plantean balances de compuestos orgánicos y nutrientes (Pepperell et al., 2011). Así mismo, los mecanismos de eliminación de nitrógeno se siguen estudiando (Zimmo et al., 2004; Shen et al., 2012) pues existen divergencias frente a cuáles son los más efectivos y cuáles son los predominantes en estos reactores (Camargo, 2008). De ahí la importancia de desarrollar modelos ecológicos en LFS para C y N. Este documento aborda una propuesta de modelado ecológico en LFS para ambos elementos.

\section{Desarrollo}

La investigación fue ejecutada en la Estación de Investigación de Aguas residuales y reúso de Ginebra, EIG, localizada en el Valle del Cauca, Colombia, a $3^{\circ} 43^{\prime} 50^{\prime \prime}$ latitud norte y $76^{\circ} 16^{\prime} 20^{\prime \prime}$ longitud este, a 1040 ms.n.m. La temperatura promedio es $23^{\circ} \mathrm{C}$ y la precipitación promedio es $1280 \mathrm{~mm}$. Se hizo seguimiento a tres LFS con distintas configuraciones físicas (tabla 1). El diseño de las unidades piloto se realizó empleando la metodología de carga orgánica superficial (Mara, 2001) aplicando un análisis de incertidumbre (Von Sperling, 1996) con base en la propuesta de Banda (2003).

La figura 2 ilustra los pilotos y los puntos de muestreo. En P1 y P2 se tomaron muestras puntuales a dos profundidades, $0.05 \mathrm{~m}$ y $0.75 \mathrm{~m}$, a las 10:00, 13:00, 17:00 y 22:00 horas teniendo en cuenta las variaciones meteorológicas; también se tomaron muestras puntuales y compuestas a la entrada y la salida de cada unidad. Se 


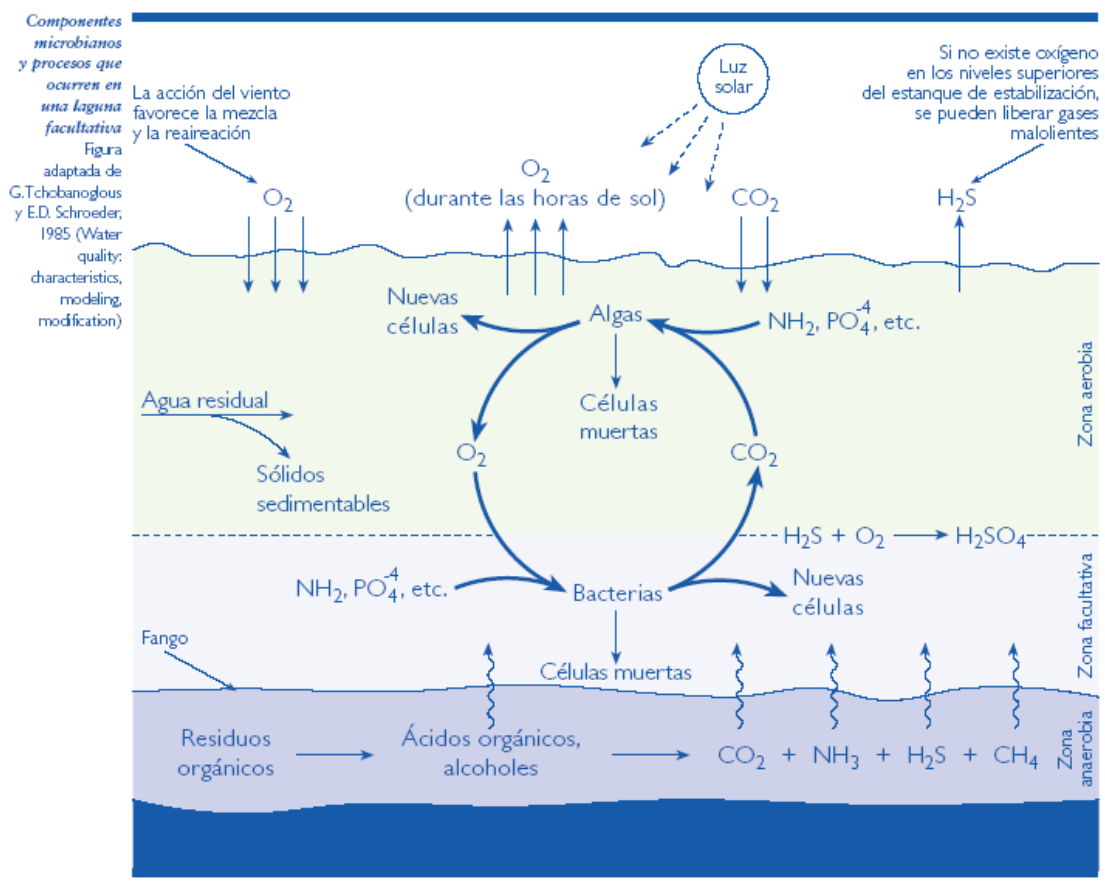

Figura 1. Ecosistema en una LFS. Fuente: Metcalf y Eddy (1995)

Tabla 1. Características de las unidades piloto

\begin{tabular}{|c|c|c|c|}
\hline Características de diseño & Piloto 1. Bafles Mallas, LBM & Piloto 2. Convencional, LC & Piloto 3. Bafles, LB \\
\hline Modificación & $\begin{array}{c}\text { Construcción bafles e instalación } \\
\text { de mallas a L/3 y } 2 / \mathrm{L} 3\end{array}$ & & $\begin{array}{c}\text { Construcción de dos bafles } \\
\text { a L/3 y } 2 / \mathrm{L} 3\end{array}$ \\
\hline Caudal (L/s) & 0.275 & 0.275 & 0.275 \\
\hline Relación L/B & 3.01 & 3.10 & 3.15 \\
\hline Altura (m) & 1.39 & 1.31 & 1.32 \\
\hline Talud X:Y & 0.99 & 0.91 & 0.91 \\
\hline Ancho espejo de agua $(\mathrm{m})^{*}$ & 5.81 & 5.64 & 5.58 \\
\hline Largo espejo de agua $(m)^{*}$ & 17.46 & 17.49 & 17.57 \\
\hline Volumen $\left(\mathrm{m}^{3}\right)^{*}$ & 99.01 & 92.01 & 94.64 \\
\hline TRH (días) ${ }^{* *}$ & 4.17 & 3.99 & 3.98 \\
\hline Área $\left(\mathrm{m}^{2}\right)$ & 45.08 & 49.25 & 48.16 \\
\hline Carga aplicada (kg DBO/Ha) & 271.23 & 278.92 & 280.64 \\
\hline
\end{tabular}

* Las diferencias responden a condiciones constructivas. Se admitieron diferencias menores a 5\% en las variables TRH, Área y Carga aplicada

** Corresponde al TRH teórico o nominal

midieron caudales en forma volumétrica a la entrada y a la salida y se realizaron pruebas físico-químicas de las variables de interés a todas las muestras recolectadas (tabla 2). Con esta información de seguimiento se obtuvo información para la validación y calibración de los modelos ecológicos propuestos.

La formulación de los modelos fue guiada por la propuesta de Jorgensen y Bendoricchio (2001) (figura
3). El diagrama conceptual se desarrolló con el software Structural Thinking Experimental Learning Laboratory with Animation, Stella, de ISEE Systems Inc. ${ }^{\circledR}$ Este software se ha utilizado para simular modelos ecológicos y ofrece ventajas desde el punto de vista gráfico y de cuantificación (Jamu y Piedrahita, 2002). Las expresiones de balance de materia para cada especie fueron la base para la formulación matemática de los 
Tabla 2. Variables medidas en las unidades piloto

\begin{tabular}{ccccc}
\hline Parámetro & Unidades & Método de medición & Lugar de medición & Ref. Standard methods \\
\hline $\mathrm{pH}$ & Unidades & Potenciómetro & In situ & $4500-\mathrm{H}^{+} \mathrm{B}$ \\
Temperatura & ${ }^{\circ} \mathrm{C}$ & Térmico & In situ & $2550 \mathrm{~B}$ \\
Oxígeno disuelto & $\mathrm{mg} / \mathrm{L}$ & Potenciómetro & In situ & $4500-0 \mathrm{G}$ \\
Potencial Redox & $\mathrm{mV}$ & Potenciómetro & In situ & $2580 \mathrm{~B}$ \\
Clorofila $a$ & $\mu \mathrm{g} / \mathrm{L}$ & Fluorometria & Laboratorio & $10200 \mathrm{H}$ \\
$\mathrm{DQO}^{*}$ & $\mathrm{mg} / \mathrm{L}$ & Digestión & Laboratorio & $5220 \mathrm{D}$ \\
$\mathrm{DBO}_{5}^{*}{ }^{* *}$ & $\mathrm{mg} / \mathrm{L}$ & Winlker & Laboratorio & $5210 \mathrm{~B}$ \\
$\mathrm{SST}^{*}$ & $\mathrm{mg} / \mathrm{L}$ & Laboratorio & $2540 \mathrm{D}$ \\
$\mathrm{N}^{*} \mathrm{NH}_{4}{ }^{+}$ & $\mathrm{mg} / \mathrm{L}$ & Labimétrico & Laboratorio & $4500-\mathrm{NH}_{3} \mathrm{~A}$ \\
$\mathrm{NTK}^{\mathrm{mg} / \mathrm{L}}$ & $\mathrm{mg} / \mathrm{L}$ & Kjeldahl & Laboratorio & $4500_{\text {-org }} \mathrm{B}$ \\
$\mathrm{N} / \mathrm{NO}_{3}{ }^{-}$ & $\mathrm{mg} / \mathrm{L}$ & Potenciómetro & & $4500-\mathrm{NO}_{3} \mathrm{D}$ \\
$\mathrm{Alcalinidad}$ & $\mathrm{mg} / \mathrm{L}$ & Combustión-infrarrojo & Laboratorio & $5310 \mathrm{~A}$ \\
$\mathrm{COT}$ & & & & \\
\hline
\end{tabular}

Fuente: APHA-AWWA-WEF (2005)

* Total y filtrada para las muestras compuestas

** Solo se midió en muestras compuestas

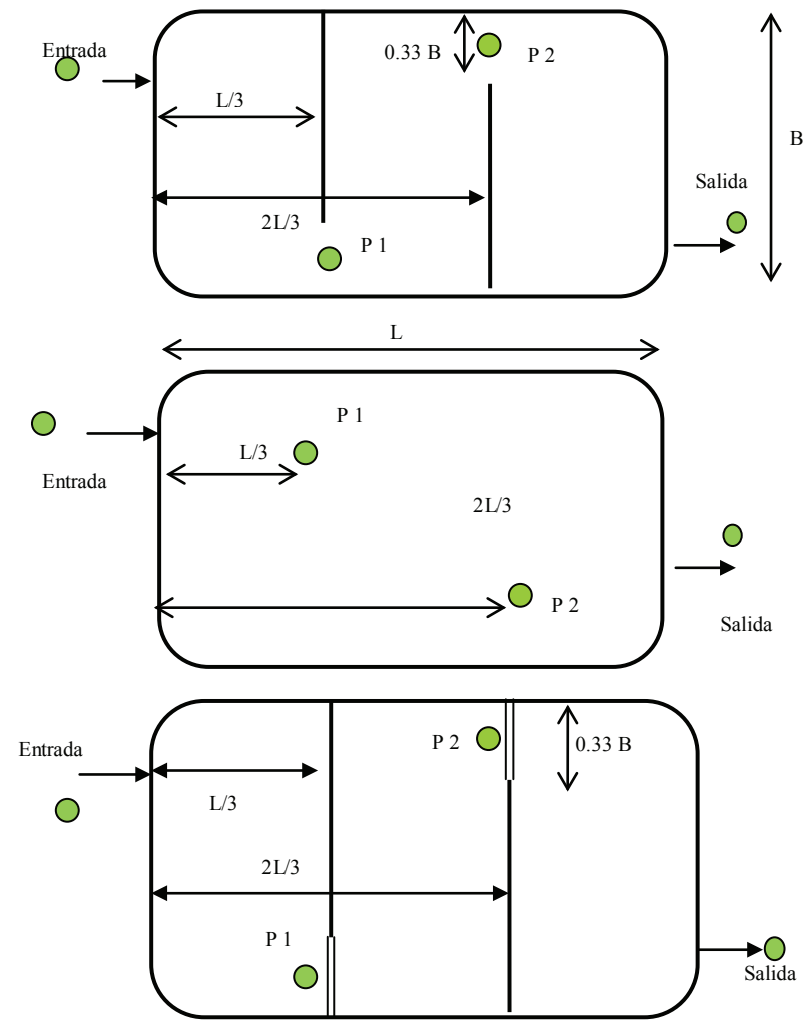

Figura 2.Esquema de unidades piloto y puntos de muestreo. De abajo arriba, LBM, LC y LB modelos ecológicos (ecuación 1). Los modelos consideraron condiciones no estacionarias, lo que definió una variación con respecto al tiempo de las especies químicas estudiadas.

$\frac{\mathrm{dM}}{\mathrm{dt}}=\mathrm{Q}_{\mathrm{i}} *\left[\mathrm{C}_{\mathrm{i}}\right]-\mathrm{Q}_{\mathrm{e}} *\left[\mathrm{C}_{\mathrm{e}}\right] \pm \mathrm{R}_{\mathrm{c}}$

donde:

$\mathrm{Rc}_{\mathrm{C}}=\mathrm{r}_{\mathrm{M}} * \mathrm{~V}\left(\mathrm{MT}^{-1}\right)$

$\mathrm{r}_{\mathrm{M}}=$ transformación de la especie de interés en la LFS (aparición o desaparición) $\left(\mathrm{ML}^{-3} \mathrm{t}^{-1}\right)$

$\mathrm{V}=$ volumen de la laguna. $\left(\mathrm{L}^{3}\right)$

$\mathrm{Q}=\operatorname{caudal}\left(\mathrm{L}^{3} \mathrm{t}^{-1}\right)$

$\left[\mathrm{C}_{\mathrm{i}}\right]=$ concentración de la especie a la entrada $\left(\mathrm{ML}^{-3}\right)$

$\left[\mathrm{C}_{\mathrm{e}}\right]=$ concentración de la especie a la salida $\left(\mathrm{ML}^{-3}\right)$

(MtCada ecuación de balance de materia para las especies de interés fue implementada en el software Stella $₫$. Se construyeron sub modelos (Pereira et al., 2006) para:

- Carbono orgánico total

- Carbono inorgánico total

- Nitrógeno orgánico particulado

- Nitrógeno orgánico disuelto

- Nitrógeno de nitratos

- Nitrógeno amoniacal 

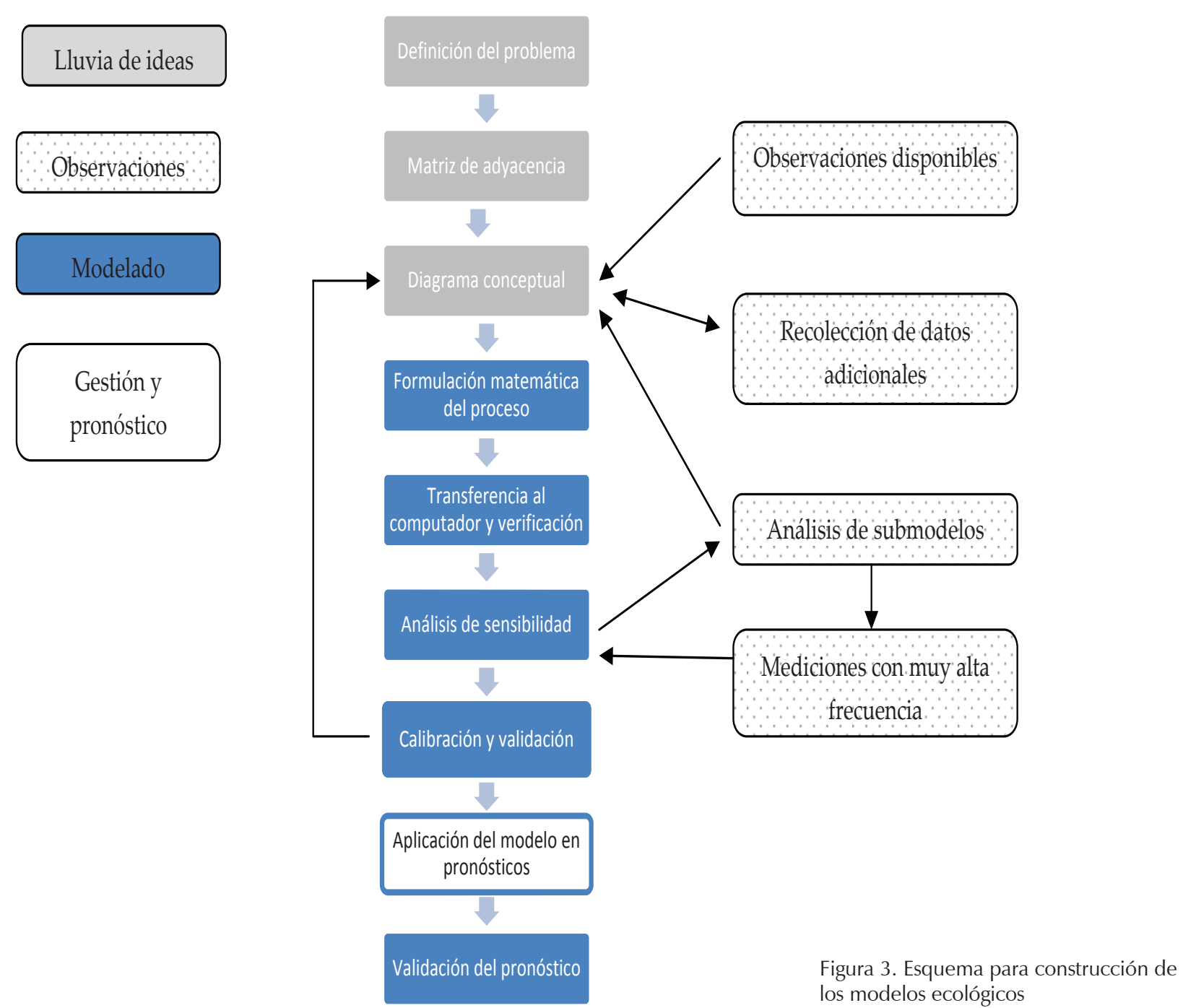

A partir de los resultados para LC se verificó el modelo correspondiente a LBM, ajustando los parámetros respectivos. El software Stella ${ }^{\circledR}$ fue utilizado en un equipo Toshiba Tecra, Procesador Intel ${ }^{\circledR}$ Core $^{\mathrm{TM}} 2$ Duo, CPU T8100@2.10 GHz 2.09 GHz 1.99 GB de RAM. El Apéndice 1 resume las expresiones matemáticas empleadas para la formulación de los modelos; la mayoría de ellas respondieron a cinéticas tipo Monod, considerando las condiciones hipertróficas de las LFS. La tabla 3 y 4 presentan los parámetros y valores empleados para ambos modelos. La LC se asumió como reactor completamente mezclado, RCM, con base en información de estudios de trazadores realizados en la LC y la LBM (Bravo y Rodriguez, 2010; Fichmann,
2012) los cuales arrojaron como resultado un comportamiento de flujo mixto con tendencia a RCM, correspondiente con lo planteado por otros investigadores (Mara, 2004), los números de dispersión hallados para LBM oscilaron entre 0.31 y 0.43 y para LC entre 0.19 y 1.5. Las concentraciones de biomasa algal y bacterial se hallaron a partir de los contenidos de COT en las lagunas piloto.

Los valores de nitrógeno asociado a microalgas y bacterias se estimaron a partir de la literatura (Mišurcová et al., 2010; Bradley y Nichols, 1918; Fagerbakke et al., 1996). En la tabla 5 se presentan las interacciones entre las distintas especies de análisis de los modelos propuestos para $\mathrm{C}$ y $\mathrm{N}$. 
Tabla 3. Parámetros modelos de Carbono

\begin{tabular}{|c|c|c|c|c|}
\hline Parámetro & Definición & Unidades & Rango & Fuente \\
\hline $\mathrm{Y}_{\text {Bha }}$ & $\begin{array}{l}\text { Relación sustrato y biomasa (bacterias } \\
\text { heterotróficas) }\end{array}$ & & 0.788 & (Mashauri y Kayombo, 2002) \\
\hline $\mathrm{X}_{\mathrm{Ba}}$ & Concentración Biomasa Bacterias & $\mathrm{g} \mathrm{N} \mathrm{m}^{-3}$ & Variable & Experimental \\
\hline $\mathrm{u}_{\max B a}$ & Tasa crecimiento máximo Bacterias & $d^{-1}$ & $3.8,2-10$ & $\begin{array}{l}\text { (Kayombo et al., 2003; } \\
\text { Tchobanoglous et al., 2004) }\end{array}$ \\
\hline COT & Carbono orgánico Total & $\mathrm{g} \mathrm{C} \mathrm{m}^{-3}$ & Variable & Experimental \\
\hline $\mathrm{K}_{\text {СОт Ва }}$ & $\begin{array}{l}\text { Constante saturación media para oxidación } \\
\text { (COT) por Bacterias }\end{array}$ & $\mathrm{g} \mathrm{C} \mathrm{m}^{-3}$ & $200,40-50$ & $\begin{array}{l}\text { (Kayombo et al., 2003; } \\
\text { Tchobanoglous et al., 2004) }\end{array}$ \\
\hline $\mathrm{K}_{\text {O2Ba }}$ & $\begin{array}{l}\text { Constante saturación media para } \mathrm{O}_{2} \text { por } \\
\text { Bacterias }\end{array}$ & $\mathrm{g} \mathrm{O}_{2} \mathrm{~m}^{-3}$ & 1 & (Fritz et al., 1979) \\
\hline $\mathrm{K}_{4 \mathrm{Ba}}$ & $\begin{array}{l}\text { Constante saturación media para nitrógeno } \\
\text { tomado por Bacterias }\end{array}$ & $\mathrm{g} \mathrm{N} \mathrm{m}^{-3}$ & 0.05 & (Henzen et al., 1995) \\
\hline $\mathrm{T}_{\text {opt Ba }}$ & Temperatura óptima crecimiento Bacterias & ${ }^{\circ} \mathrm{C}$ & $20-50$ & (Tortora et al., 2007) \\
\hline $\mathrm{Tx}_{\mathrm{Ba}}$ & Temperatura para crecimiento bacterias & ${ }^{\circ} \mathrm{C}$ & $0-15$ & Ídem \\
\hline $\mathrm{Opt}_{\mathrm{pHBa}}$ & pH óptimo para crecimiento Bacterias & & $6-11$ & (Kayombo et al., 2000) \\
\hline $\mathrm{K}_{\mathrm{pHBa}}$ & Constante media de velocidad para $\mathrm{pH}$ & & $150-250$ & Ídem \\
\hline $\mathrm{K}_{\mathrm{e}}$ & Coeficiente de extinción de luz & $\mathrm{m}^{-1}$ & 13 & (Heaven y Zotova, 2005) \\
\hline$I_{S}$ & Radiación solar en superficie & Langley d ${ }^{-1}$ & $0-596.6$ & Experimental \\
\hline $\mathrm{D}$ & Profundidad para la intensidad de luz & $\mathrm{m}$ & 0.18 & Experimental \\
\hline$I_{k}$ & Intensidad óptima & Langley $\mathrm{d}^{-1}$ & 250,450 & $\begin{array}{l}\text { (Fritz et al., 1979; Beran and } \\
\text { Kargi, } 2005\end{array}$ \\
\hline $\mathrm{X}_{\mathrm{Al}}$ & Concentración de biomasa algal & $\mathrm{g} \mathrm{N} \mathrm{m}^{-3}$ & & Experimental \\
\hline $\mathrm{u}_{\max \mathrm{Al}}$ & Tasa de crecimiento Microalgas & $\mathrm{d}^{-1}$ & $0.5-2,2$ & $\begin{array}{l}\text { (Asaeda y Van, 1997; Sah, } \\
\text { 2009) }\end{array}$ \\
\hline $\mathrm{T}_{\mathrm{optAl}}$ & Temperatura óptima crecimiento microalgas & ${ }^{\circ} \mathrm{C}$ & 25 & (Muñoz y Guieysse, 2006) \\
\hline $\mathrm{Tx}_{\mathrm{Al}}$ & Temperatura máxima crecimiento Algas & ${ }^{\circ} \mathrm{C}$ & 40 & Ídem \\
\hline $\mathrm{CO}_{2 \mathrm{~d}}$ & Concentración de $\mathrm{CO}_{2}$ en el día & $\mathrm{g} \mathrm{CO}_{2} \mathrm{~m}^{-3}$ & Calibración & Experimental \\
\hline $\mathrm{K}_{\mathrm{CO} 2 \mathrm{Al}}$ & $\begin{array}{l}\text { Constante de saturación media } \mathrm{CO} 2 \text { tomado } \\
\text { por microalgas }\end{array}$ & $\mathrm{g} \mathrm{CO}_{2} \mathrm{~m}^{-3}$ & $1,0.5-0.6$ & $\begin{array}{l}\text { (Fritz et al., 1979; Chen y } \\
\text { Orlob, 1975) }\end{array}$ \\
\hline $\mathrm{K}_{\mathrm{NAl}}$ & $\begin{array}{l}\text { Constante de saturación media para } \\
\text { nitrógeno tomado por microalgas }\end{array}$ & $\mathrm{g} \mathrm{N} \mathrm{m}^{-3}$ & 0.025 & (Beran y Kargi, 2005) \\
\hline $\mathrm{Opt}_{\mathrm{pHAl}}$ & pH óptimo para crecimiento microalgas & & $6-11$ & (Kayombo et al., 2000) \\
\hline $\mathrm{K}_{\mathrm{pHAl}}$ & Constante media de velocidad & & $150-250$ & Ídem \\
\hline $\mathrm{K}_{10 \mathrm{x}}$ & Tasa de crecimiento & $d^{-1}$ & $0.1-1.2$ & (Reed et al., 1998) \\
\hline $\mathrm{K}_{1 \mathrm{O} 2}$ & $\begin{array}{l}\text { Constante saturación media para oxígeno } \\
\text { disuelto en la respiración aerobia }\end{array}$ & $\mathrm{g} \mathrm{O}_{2} \mathrm{~m}^{-3}$ & $0.1-1$ & (Tchobanoglous et al., 2004) \\
\hline$\Theta_{10 x}$ & $\begin{array}{l}\text { Coeficiente temperatura para respiración } \\
\text { aerobia }\end{array}$ & & $0.967-1.104$ & (Mashauri y Kayombo, 2002) \\
\hline $\mathrm{V}_{\mathrm{Al}}$ & Velocidad sedimentación Algas & $\mathrm{m} \mathrm{d}^{-1}$ & $0.27-0.89$ & $\begin{array}{l}\text { (Jorgensen y Bendoricchio, } \\
\text { 2001) }\end{array}$ \\
\hline $\mathrm{V}_{\mathrm{Ba}}$ & Velocidad sedimentación Bacterias & $\mathrm{m} \mathrm{d}^{-1}$ & $0.005-0.5$ & (Mashauri y Kayombo, 2002) \\
\hline h & Profundidad laguna & $\mathrm{m}$ & 1.31 & Experimental \\
\hline V & Volumen laguna & $\mathrm{m}^{3}$ & 92.01 & Experimental \\
\hline $\mathrm{K}_{\mathrm{al}}$ & Coeficiente de respiración microalgas & & 0.08 & (Fritz et al., 1979) \\
\hline $\mathrm{K}_{1 \mathrm{D}}$ & Constante de saturación media del $\mathrm{CO}_{2}$ & $d^{-1}$ & $0.9664-0.0208^{*} \mathrm{~T}$ & (Beran y Kargi, 2005) \\
\hline A & Área laguna & $\mathrm{m}^{2}$ & 98.64 & Experimental \\
\hline $\mathrm{K}_{\mathrm{O} 2}$ & Constante de saturación media del $\mathrm{O}_{2}$ & & $\begin{array}{l}\left(0.728 \mathrm{~W}_{\mathrm{A}-\mathrm{LQ25}} \cdot 0.31 \mathrm{~W}_{A-L}+\right. \\
\left.0.0372 \mathrm{~W}_{A-L}\right)(1.0241(\mathrm{~T}- \\
20))\end{array}$ & (Beran y Kargi, 2005) \\
\hline $\mathrm{W}_{\mathrm{A}-\mathrm{L}}$ & Velocidad del viento sobre la laguna & $\mathrm{m} \mathrm{s}^{-1}$ & Variable & Experimental \\
\hline $\mathrm{W}_{\mathrm{L}-\mathrm{A}}$ & $\begin{array}{l}\text { Velocidad del viento sobre la superficie de } \\
\text { la laguna }\end{array}$ & $\mathrm{m} \mathrm{s}^{-1}$ & Variable & Experimental \\
\hline $\mathrm{R}_{\mathrm{DBe}}$ & Tasa difusión del bentos $\mathrm{CO}_{2}$ & $\mathrm{gC} \mathrm{d}^{-1}$ & 0.09 & \\
\hline $\mathrm{R}_{\mathrm{CH} 4 \mathrm{Be}}$ & Tasa difusión del bentos $\mathrm{CH}_{4}$ & $\mathrm{gC} \mathrm{d}^{-1}$ & Calibración & Experimental \\
\hline $\mathrm{V}_{\mathrm{b}}$ & Volumen del bentos & $\mathrm{m}^{3}$ & Calibración & Experimental \\
\hline $\mathrm{R}_{1 \mathrm{DCH} 4}$ & $\begin{array}{l}\text { Tasa volatilización } \mathrm{CH}_{4} \text { desde la columna } \\
\text { de agua }\end{array}$ & $\mathrm{gC} \mathrm{d}^{-1}$ & 162.756 & (Lasso, 2010) \\
\hline
\end{tabular}


Tabla 4. Parámetros modelos de Nitrógeno

\begin{tabular}{|c|c|c|c|c|}
\hline Parámetros & Definición & Unidades & Rango & Fuente \\
\hline $\mathrm{K}_{\text {Den }}$ & Tasa velocidad denitrificación & $\mathrm{d}^{-1}$ & 0.1 & (Chao et al., 2007) \\
\hline $\begin{array}{l}\theta_{\text {Den }} \\
V\end{array}$ & $\begin{array}{l}\text { Coeficiente temperatura } \\
\text { Velocidad sedimentación algas }\end{array}$ & $\mathrm{m} \mathrm{d}^{-1}$ & $\begin{array}{c}1.045 \\
0.27-0.89\end{array}$ & $\begin{array}{l}\text { (Jorgensen y Bendoricchio, 2001) } \\
\text { Ídem }\end{array}$ \\
\hline $\mathrm{V}_{\mathrm{Ba}}$ & Velocidad sedimentación bacterias & $\mathrm{m} \mathrm{d}^{-1}$ & $0.005-0.5$ & $\begin{array}{l}\text { (Mashauri y Kayombo, 2002; } \\
\text { Farrás, 2005) }\end{array}$ \\
\hline $\mathrm{h}$ & Profundidad laguna & $\mathrm{m}$ & 1.31 & Experimental \\
\hline $\mathrm{K}_{\mathrm{O} 2 \mathrm{~N}}$ & $\begin{array}{l}\text { Constante saturación media para el oxígeno en } \\
\text { la nitrificación }\end{array}$ & $\mathrm{g} \mathrm{O}_{2} \mathrm{~m}^{-3}$ & $1.3-0.5$ & $\begin{array}{l}\text { (Fritz et al., } 1979 \text { Henzen et al, } \\
\text { 1995) }\end{array}$ \\
\hline $\mathrm{K}_{4 \mathrm{~N}}$ & $\begin{array}{l}\text { Constante saturación media para amonio en la } \\
\text { nitrificación }\end{array}$ & $\mathrm{g} \mathrm{N} \mathrm{m}^{-3}$ & 0.025 & \\
\hline $\mathrm{u}_{\mathrm{N}}$ & Tasa crecimiento máximo nitrosomonas & $\mathrm{d}^{-1}$ & 0.008 & (Fritz et al., 1970) \\
\hline$Y_{N}$ & Factor crecimiento nitrosomonas & & 0.15 & İdem \\
\hline $\mathrm{C}_{\mathrm{pH}}$ & $\begin{array}{l}\text { Factor inhibición para crecimiento } \\
\text { nitrosomonas por } \mathrm{pH}\end{array}$ & & $\left(1-0.8333^{*}(7.2-\mathrm{pH})\right)$ & Ídem \\
\hline $\mathrm{K}_{\mathrm{ODA}}$ & Tasa amonificación NOD & $\mathrm{d}^{-1}$ & 0.1 & (Qitao y Youngchul, 200) \\
\hline$\theta_{\mathrm{ODA}}$ & Coeficiente temperatura amonificación NOD & & 1.02 & $\begin{array}{l}\text { (Jorgensen y Bendoricchio, } \\
\text { 2001) }\end{array}$ \\
\hline $\mathrm{K}_{\mathrm{OPH}}$ & Tasa velocidad hidrólisis NOP & $\mathrm{d}^{-1}$ & $0.001-0.01$ & (Qitao y Youngchul, 2009) \\
\hline $\mathrm{K}_{\mathrm{OPA}}$ & Tasa amonificación NOP & $\mathrm{d}^{-1}$ & $0.01-0.03$ & $\begin{array}{l}\text { (Jorgensen y Bendoricchio, } \\
\text { 2001) }\end{array}$ \\
\hline$\theta_{\mathrm{OPA}}$ & Coeficiente temperatura amonificación NOP & & $1.02-1.08$ & Ídem \\
\hline $\mathrm{X}_{\mathrm{Ba}}$ & Concentración biomasa bacterias & $\mathrm{g} \mathrm{N} / \mathrm{m}^{3}$ & Variable & Experimental \\
\hline $\mathrm{u}_{\max B a}$ & Tasa crecimiento máximo bacterias & $\mathrm{d}^{-1}$ & $3.8,2-10$ & $\begin{array}{l}\text { (Kayombo et al., 2003; } \\
\text { Tchobanoglous et al., 2004) }\end{array}$ \\
\hline COT & Carbono orgánico total & $\mathrm{gC} \mathrm{m}^{-3}$ & Variable & Experimental \\
\hline $\mathrm{K}_{\text {СОт Ва }}$ & $\begin{array}{l}\text { Constante saturación media oxidación (COT) } \\
\text { por bacterias }\end{array}$ & $\mathrm{g} \mathrm{C} \mathrm{m}^{-3}$ & $200,40-50$ & $\begin{array}{l}\text { (Kayombo et al., 2003; } \\
\text { Tchobanoglous et al., 2004) }\end{array}$ \\
\hline $\mathrm{K}_{\mathrm{O} 2 \mathrm{Ba}}$ & Constante saturación media $\mathrm{O}_{2}$ por bacterias & $\mathrm{g} \mathrm{O}_{2} \mathrm{~m}^{-3}$ & 1 & (Fritz et al., 1979) \\
\hline $\mathrm{K}_{4 \mathrm{Ba}}$ & $\begin{array}{l}\text { Constante saturación media nitrógeno tomado } \\
\text { por bacterias }\end{array}$ & $\mathrm{g} \mathrm{N} \mathrm{m}^{-3}$ & 0.05 & (Henzen et al., 1995) \\
\hline $\mathrm{T}_{\text {opt Ba }}$ & Temperatura óptima crecimiento bacterias & ${ }^{\circ} \mathrm{C}$ & $20-50$ & (Tortora et al., 2007) \\
\hline $\mathrm{T}_{\mathrm{xBa}}$ & Temperatura mínima crecimiento bacterias & ${ }^{\circ} \mathrm{C}$ & $0-15$ & Ídem \\
\hline $\mathrm{Opt}_{\mathrm{pHBa}}$ & pH óptimo crecimiento bacterias & & 6-11 & (Kayombo et al., 2000) \\
\hline $\mathrm{K}_{\mathrm{pHBa}}^{\mathrm{pHBa}}$ & Constante media de velocidad & & $150-250$ & Ídem \\
\hline & Coeficiente de extinción de luz & $\mathrm{m}^{-1}$ & 13 & (Heaven y Zotova, 2005) \\
\hline $\mathrm{I}_{S}{ }^{e}$ & Radiación solar en superficie & Langley $\mathrm{d}^{-1}$ & $0-596.6$ & Experimental \\
\hline $\mathrm{D}$ & Profundidad para la intensidad de luz & $\mathrm{m}$ & 0.18 & Experimental \\
\hline$I_{k}$ & Intensidad óptima & Langley $\mathrm{d}^{-1}$ & 250,450 & $\begin{array}{l}\text { (Fritz et al., 1979; Beran y Kargi, } \\
\text { 2005) }\end{array}$ \\
\hline $\mathrm{X}_{\mathrm{Al}}$ & Concentración biomasa algal & $\mathrm{g} \mathrm{N} \mathrm{m}^{-3}$ & Variable & Experimental \\
\hline $\mathrm{u}_{\text {máx Al }}$ & Tasa crecimiento para microalgas & $\mathrm{d}^{-1}$ & $0.5-2,2$ & (Asaeda y Van, 1997; Sah, 2009) \\
\hline $\mathrm{T}_{\text {opt Al }}$ & Temperatura óptima crecimiento microalgas & ${ }^{\circ} \mathrm{C}$ & 25 & (Muñoz y Guieysse, 2006) \\
\hline $\operatorname{Tx}_{\mathrm{Al}}$ & Temperatura máxima crecimiento microalgas & ${ }^{\circ} \mathrm{C}$ & 40 & Ídem \\
\hline $\mathrm{CO}_{2}$ & Concentración $\mathrm{CO}_{2}$ & $\mathrm{~g} \mathrm{CO}_{2} \mathrm{~m}^{-3}$ & Variable & Experimental \\
\hline $\mathrm{KC}_{\mathrm{O} 2 \mathrm{Al}}$ & $\begin{array}{l}\text { Constante saturación media } \mathrm{CO}_{2} \text { tomado por } \\
\text { microalgas }\end{array}$ & $\mathrm{g} \mathrm{CO}_{2} \mathrm{~m}^{-3}$ & $1,0.5-0.6$ & $\begin{array}{l}\text { (Fritz et al., 1979, Chen y Orlob, } \\
\text { 1975) }\end{array}$ \\
\hline $\mathrm{K}_{\mathrm{NAl}}$ & $\begin{array}{l}\text { Constante saturación media nitrógeno tomado } \\
\text { por microalgas }\end{array}$ & $\mathrm{g} \mathrm{N} \mathrm{m}^{-3}$ & 0.025 & (Beran y Kargi, 2005) \\
\hline $\mathrm{Opt}_{\text {pHAl }}$ & pH óptimo crecimiento microalgas & Un & 6-11 & (Kayombo et al., 2000) \\
\hline $\mathrm{K}_{\mathrm{pHAl}}$ & Constante media de velocidad & & $150-250$ & Ídem \\
\hline $\mathrm{K}_{4 \mathrm{~V}}$ & $\begin{array}{l}\text { Coeficiente transferencia de masa en la fase } \\
\text { líquida }\end{array}$ & $d^{-1}$ & $\left.(0.0566 / \mathrm{h})^{*}\left(\mathrm{e}^{\left(0.13^{*}(\mathrm{~T}-20)\right.}\right)\right)$ & (Zimmo et al., 2003) \\
\hline $\mathrm{NH}_{4}^{+}$ & $\begin{array}{l}\text { Concentración amonio libre en columna de } \\
\text { agua }\end{array}$ & $\mathrm{g} \mathrm{N} \mathrm{m}^{-3}$ & & \\
\hline A & Porcentaje $\mathrm{NH}_{4}^{+}$presente en columna de agua & & Variable & (Camargo, 2008) \\
\hline $\mathrm{pk}_{\mathrm{b}}$ & Constante equilibrio disociación de $\mathrm{NH}_{4}^{+}$ & & $\begin{array}{c}0.09018 \\
+(2729.92 /(273.2+\mathrm{T}))\end{array}$ & Ídem \\
\hline $\mathrm{T}_{\text {AmoBen }}$ & Tasa amonificación NOP bentos & & Calibración & Experimental \\
\hline $\mathrm{V}$ & Volumen laguna & $\mathrm{m}^{3}$ & 90.12 & Experimental \\
\hline $\mathrm{r}_{4 \mathrm{An}}$ & Tasa anammox & $\mathrm{g} \mathrm{N} \mathrm{m}^{-3} \mathrm{~d}^{-1}$ & 0.000802 & (Van et al., 2010) \\
\hline
\end{tabular}


DOI: https://doi.org/10.1016/S1405-7743(14)70353-1

Desarrollo de modelos ecológicos para carbono y nitrógeno en lagunas facultativas secundarias

Tabla 5. Matriz de adyacencia

\begin{tabular}{|c|c|c|c|c|c|c|c|}
\hline Variable & Definición & COT & $\mathrm{CO}_{2}$ & $\mathrm{NH}_{4}^{+}$ & $\mathrm{NO}_{3}^{-}$ & NOP & NOD \\
\hline$R_{1 R a n}$ & Tasa consumo carbono orgánico por anaerobiosis & $\mathrm{X}$ & $\mathrm{X}$ & & & & \\
\hline$R_{1 \text { Rae }}$ & Tasa consumo carbono orgánico aerobiosis & $\mathrm{X}$ & $\mathrm{X}$ & & & & \\
\hline$R_{1 \text { Ras }}$ & Tasa consumo carbono orgánico asimilación & $\mathrm{X}$ & & & & & \\
\hline$R_{1 C A}$ & Tasa producción carbono orgánico microalgas & $\mathrm{X}$ & & $\mathrm{X}$ & $\mathrm{X}$ & & \\
\hline$R_{1 C B}$ & Tasa producción carbono orgánico aerobiosis & $\mathrm{X}$ & & $\mathrm{X}$ & $\mathrm{X}$ & $\mathrm{X}$ & \\
\hline$R_{1 C B A}$ & Tasa producción carbono orgánico anaerobiosis & $\mathrm{X}$ & & $\mathrm{X}$ & & $\mathrm{X}$ & \\
\hline$R D_{C H A B S}$ & Tasa transferencia metano bentos & $\mathrm{X}$ & & & & & \\
\hline$R V_{1 S}, R_{O P S}$ & Tasa sedimentación biomasa (COP, NOP) & $\mathrm{X}$ & & & & $\mathrm{X}$ & \\
\hline$R D_{1 C H 4}$ & Tasa volatilización metano & $\mathrm{X}$ & & & & & \\
\hline$R_{F o}$ & Fotosíntesis & & $\mathrm{X}$ & & & & \\
\hline$R_{D B e}$ & Liberación desde el bentos $\mathrm{CO}_{2}$ & & $\mathrm{X}$ & & & & \\
\hline$R_{D A-L}$ & Transferencia atmósfera - columna agua $\mathrm{CO}_{2}$ & & $\mathrm{X}$ & & & & \\
\hline$R_{D L-A}$ & Transferencia columna agua - atmósfera $\mathrm{CO}_{2}$ & & $\mathrm{X}$ & & & & \\
\hline$R_{A l k}$ & Transformación $\mathrm{CO}_{2}$ a carbonatos & & $\mathrm{X}$ & & & & \\
\hline$R_{A}, R_{O P A}, R_{O D A}$ & Amonificación NOP y NOD & & & $\mathrm{X}$ & $\mathrm{X}$ & $\mathrm{X}$ & $\mathrm{X}$ \\
\hline$R_{4 N}$ & Nitrificación & & & $\mathrm{X}$ & $\mathrm{X}$ & & \\
\hline$R_{4 A n}$ & Annamox & & & $\mathrm{X}$ & & & \\
\hline$R_{4 V}$ & Volatilización $\mathrm{CH}_{4}$ & & & $\mathrm{X}$ & & & \\
\hline$R_{3 D}$ & Nitrificación & & & & $\mathrm{X}$ & & \\
\hline$R_{O P H}$ & Hidrólisis NOP & & & & & $\mathrm{X}$ & $\mathrm{X}$ \\
\hline
\end{tabular}

Los diagramas conceptuales para los modelos de $\mathrm{C}$ y $\mathrm{N}$, se presentan en las figuras 4 y 5 .

\section{Análisis y discusión}

Tabla 6. Resumen de estadísticas descriptivas de entrada de agua a los sistemas. Muestras puntuales

\begin{tabular}{|c|c|c|c|c|c|c|}
\hline Variable & Máximo & Mínimo & Promedio & SD & $\mathrm{CV}$ & $\mathrm{n}$ \\
\hline $\mathrm{pH}$ & 7,71 & 6,16 & -- & -- & -- & 32 \\
\hline TEMP AGUA $\quad\left({ }^{\circ} \mathrm{C}\right)$ & 27,90 & 23,90 & 25,78 & 1,25 & 0,05 & 32 \\
\hline OXÍGENO DISUELTO $\left(\mathrm{mgL}^{-1}\right)$ & 1,14 & 0,07 & 0,63 & 0,32 & 0,51 & 32 \\
\hline DQO TOTAL $\left(\mathrm{mgL}^{-1}\right)$ & 562,01 & 198,39 & 322,82 & 73,17 & 0,23 & 32 \\
\hline $\mathrm{SST} \quad\left(\mathrm{mgL}^{-1}\right)$ & 260,00 & 20,00 & 77,94 & 53,85 & 0,69 & 32 \\
\hline NITRATOS (NO3) $\left(\mathrm{mgL}^{-1}\right)$ & 3,99 & 0,01 & 1,14 & 1,17 & 1,03 & 32 \\
\hline NITROGENO TOTAL KJELDAHL (NTK) $\left(\mathrm{mgL}^{-1}\right)$ & 55,91 & 28,22 & 39,83 & 6,26 & 0,16 & 32 \\
\hline NITROGENO AMONIACAL (NH4+) $\left(\mathrm{mgL}^{-1}\right)$ & 38,64 & 19,99 & 29,81 & 5,05 & 0,17 & 32 \\
\hline CLOROFILA a $\left(\mu \mathrm{gL}^{-1}\right)$ & 697,03 & 26,72 & 142,39 & 129,55 & 0,91 & 32 \\
\hline $\mathrm{COT}\left(\mathrm{mgL}^{-1}\right)$ & 81,90 & 18,39 & 55,45 & 13,99 & 0,25 & 32 \\
\hline
\end{tabular}




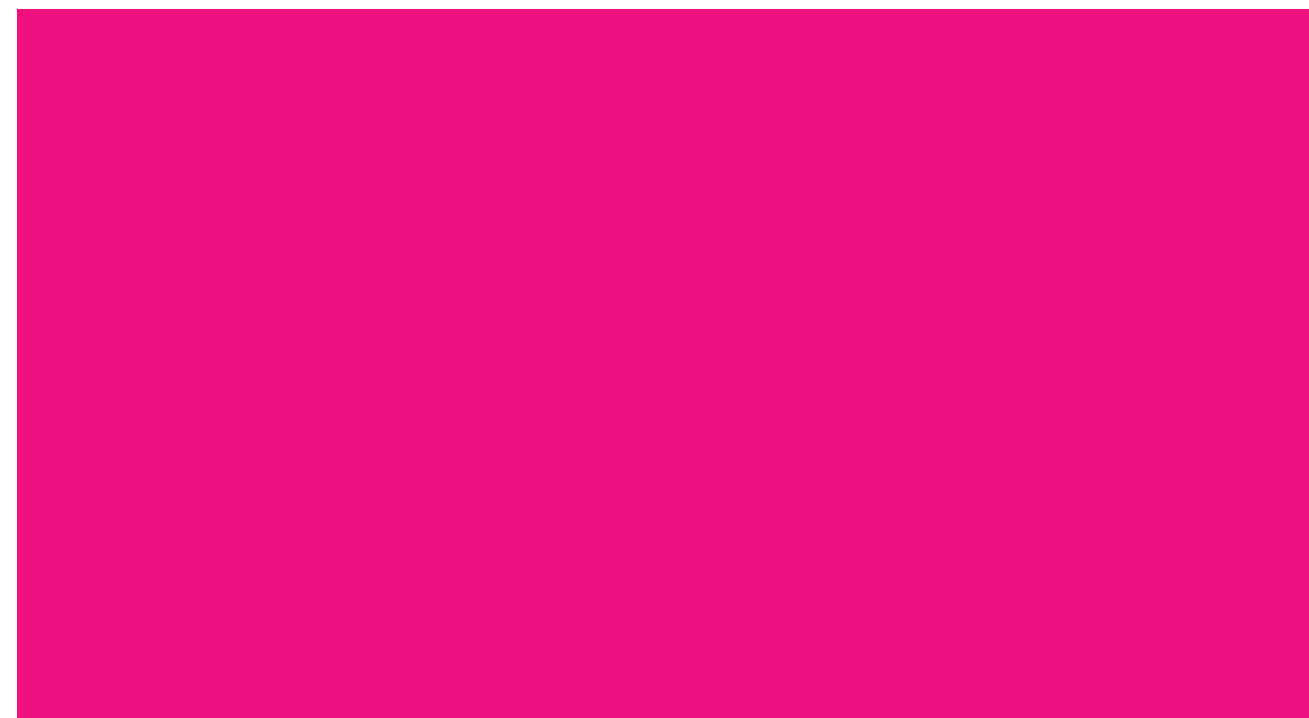

Figura 4. Diagrama conceptual modelo carbono

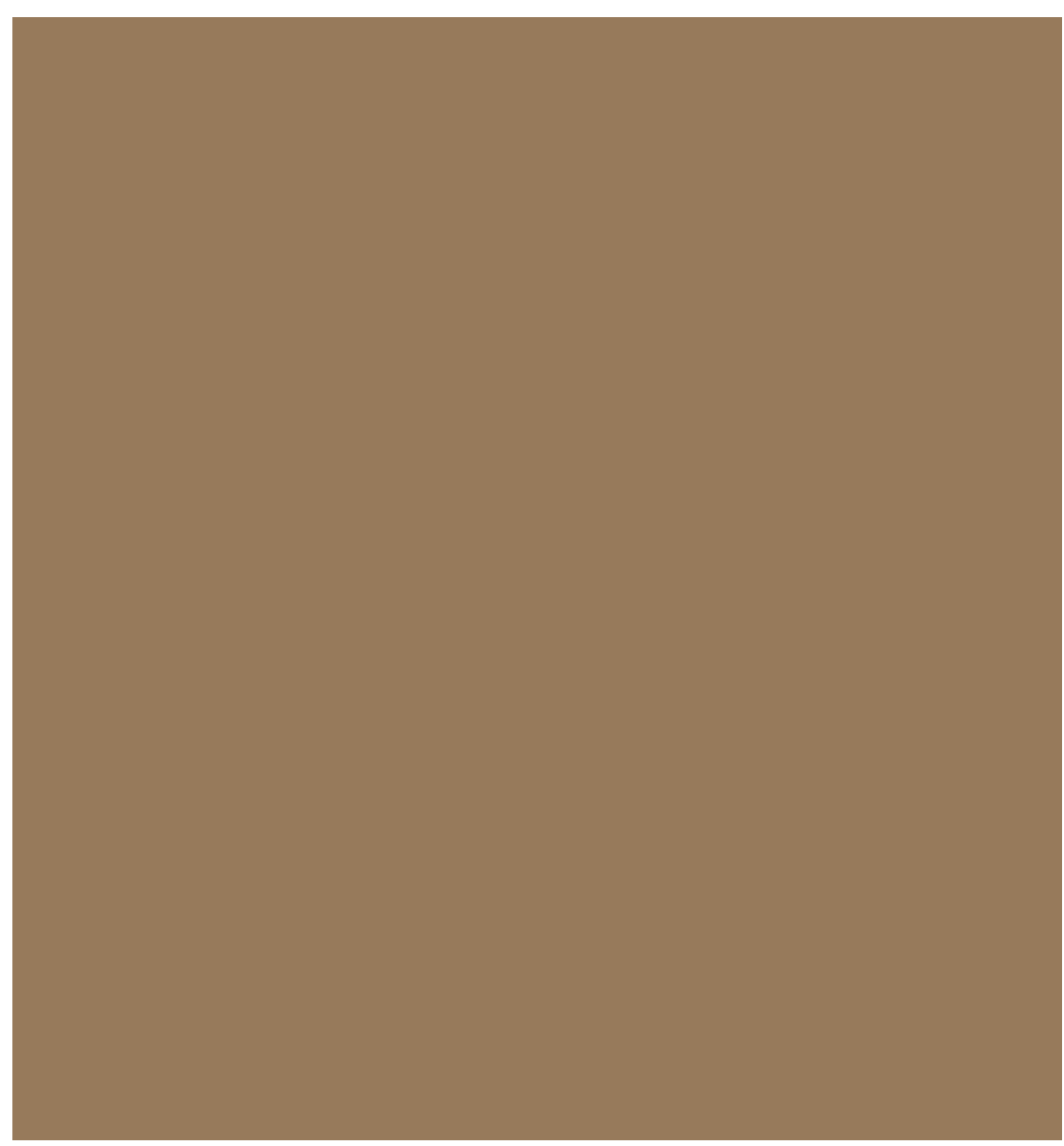

Figura 5. Diagrama conceptual modelo nitrógeno 
Las siguientes tablas ilustran las diferencias encontradas entre las unidades piloto. Las pruebas estadísticas fueron adelantadas usando el software SPSS ${ }^{\circledR}$ v. 13.0 for Windows ${ }^{\circledR}$.

\begin{tabular}{|c|c|c|c|}
\hline Variable & $\mathrm{BM} / \mathrm{C}$ & $\mathrm{BM} / \mathrm{B}$ & C / B \\
\hline DQO T & 0.055 & 0.076 & 0.425 \\
\hline SST & 0.000 & 0.002 & 0.176 \\
\hline $\mathrm{NO}_{3}{ }^{-}$ & 0.159 & 0.356 & 0.257 \\
\hline NTK & 0.055 & 0.315 & 0.114 \\
\hline $\mathrm{NH}_{4}^{+}$ & 0.022 & 0.489 & 0.033 \\
\hline Clorofila a & 0.385 & 0.266 & 0.187 \\
\hline COT & 0.273 & 0.199 & 0.392 \\
\hline
\end{tabular}

Se realizaron pruebas de normalidad para los datos y se definieron las pruebas estadísticas a utilizar. Se emplearon pruebas t-pareada para establecer diferencias en términos de gradientes horizontales y verticales (tablas $9,10$ y 11$)$.

Tabla 8. Estadísticas descriptivas Salida pilotos. Muestras puntuales

\begin{tabular}{cccc}
\hline Variable $\left(\left(\mathrm{mgL}^{-1}\right)\right)$ & Estadígrafo & BM & C \\
\hline \multirow{3}{*}{ DQO Total } & Media & 266.53 & 298.83 \\
& SD & 98.23 & 108.96 \\
& $\mathrm{n}$ & 54.00 & 53.00 \\
SST & Media & 39.51 & 61.72 \\
& $\mathrm{SD}$ & 23.46 & 36.58 \\
& $\mathrm{n}$ & 57.00 & 57.00 \\
Nitratos $\left(\mathrm{NO}_{3}{ }^{-}\right)$ & Media & 2.18 & 3.81 \\
& $\mathrm{SD}$ & 4.38 & 10.18 \\
Nitrógeno total & $\mathrm{n}$ & 47.00 & 48.00 \\
Kjeldahl $\left(\mathrm{NTK}^{\prime}\right)$ & Media & 29.79 & 28.24 \\
& $\mathrm{SD}$ & 5.21 & 4.49 \\
Nitrógeno & $\mathrm{n}$ & 50.00 & 50.00 \\
amoniacal $\left(\mathrm{NH}_{4}^{+}\right)$ & Media & 23.96 & 22.35 \\
& $\mathrm{SD}$ & 4.28 & 4.86 \\
Clorofila $a(\mu g / l)$ & $\mathrm{n}$ & 51.00 & 51.00 \\
& Media & 941.50 & 910.55 \\
& $\mathrm{SD}$ & 650.39 & 665.87 \\
COT & $\mathrm{n}$ & 77.00 & 77.00 \\
& Media & 42.06 & 43.21 \\
& $\mathrm{SD}$ & 9.01 & 9.62 \\
& $\mathrm{n}$ & 48.00 & 48.00 \\
\hline
\end{tabular}

Lo anterior indica que existieron condiciones distintas de transformación de $\mathrm{C}$ y $\mathrm{N}$ en las unidades teniendo en cuenta las variaciones identificadas entre los puntos cercanos a la salida y las salidas (P21/Sal, P22/Sal). Encontrar gradientes de Clorofila a como indicador de la presencia de microalgas en el caso de la LBM sugiere que la transformación de los compuestos de $\mathrm{C}$ y $\mathrm{N}$ está ligado a la fotosíntesis; esta aseveración coincide con los gradientes de microalgas identificados en otros ejer- cicios de modelado (Beran y Kargi, 2005; Sah, 2009). El uso del modelo de RCM quedó verificado para LC y para LBM, en el sentido de suponer disponibilidad de recursos de manera homogénea; sin embargo, los bafles y las mallas de las LBM promueven una mayor compartimentación de los procesos, como lo encontrado en el modelado hidrodinámico de lagunas con bafles que indican tendencia a Flujo Pistón, (Shilton y Harrison, 2003; Sah, 2009; Abbas et al., 2006).

Verificación de los modelos de Carbono y Nitrógeno en LC

Los modelos se consideraron verificados si para alguno de los parámetros la prueba $t$-pareada era positiva (aceptación de hipótesis nula) o si los errores entre las estimaciones y los valores de campo fueron inferiores a $50 \%$. Las etapas de análisis de sensibilidad y calibración del modelo dieron respuesta a que las pruebas $t$-pareada para todas las variables en estudio resultaran en la aceptación de la hipótesis nula. Para la verificación (tabla 11), se definieron las siguientes condiciones de configuración en el software Stella (isse-Systems, 2005).

Periodo simulación, t. 25 días.

Paso temporal, dt. 1/40.

Método de integración de las ecuaciones. método Runge-Kutta (isse-Systems, 2005).

Unidad de medida, u. Días.

La tabla 12 presenta los resultados obtenidos en el proceso de verificación. Las pruebas estadísticas indican que los datos se distribuyeron de forma normal y que los valores de COT del modelo (tabla 13) fueron estadísticamente iguales a los medidos en campo. Dado que los promedios de las diferencias entre los valores simulados y los valores medidos para las variables $\mathrm{CO}_{2}$, COT y NTK fueron inferiores a $50 \%$, a excepción de las variables $\mathrm{NH}_{4}^{+}$y $\mathrm{NO}_{3}{ }_{3}$, se consideró que los modelos quedaron verificados. Las etapas de análisis de sensibilidad y calibración permitirían ajustar los modelos para las variables $\mathrm{CO}_{2}, \mathrm{NO}_{3}{ }^{-}, \mathrm{NH}_{4}^{+}$y NTK; solo se presenta el ejercicio para COT y $\mathrm{NH}_{4}^{+}$.

Se hicieron ajustes al modelo de nitrógeno, pues los valores obtenidos para $\mathrm{NH}_{4}^{-}$no fueron satisfactorios y contrastaron con cerca de $60 \%$ que representa en el NTK según datos de campo. Durante el análisis de sensibilidad se modificó el modelo articulando en el modelo las formas de nitrógeno particulado con las de $\mathrm{NH}_{4}^{-} \mathrm{y}$ $\mathrm{NO}_{3}^{-}$como generadores de biomasa (figura 6). 
Tabla 9. Comparación entre puntos internos y entre salida LBM. Pruebas t-pareada

\begin{tabular}{|c|c|c|c|c|c|c|c|c|c|c|}
\hline & $1.1 / 1.2$ & $1.1 / 2.1$ & $1.2 / 2.1$ & $1.2 / 2.2$ & $2.1 / 2.2$ & 2.1/Sal & 2.2/Sal & $\mathrm{n}$ & G.V. & G.H. \\
\hline DQO Total & 0.120 & 0.446 & 0.161 & 0.357 & 0.168 & 0.416 & 0.113 & 24 & $\mathrm{NO}$ & $\mathrm{NO}$ \\
\hline SST Total & 0.281 & 0.396 & 0.416 & 0.252 & 0.232 & 0.029 & 0.007 & 24 & $\mathrm{NO}$ & $\mathrm{NO}$ \\
\hline $\mathrm{NO}_{3}^{-}$ & 0.051 & 0.439 & 0.423 & 0.105 & 0.063 & 0.293 & 0.232 & 18 & $\mathrm{NO}$ & $\mathrm{NO}$ \\
\hline NTK Total & 0.280 & 0.199 & 0.404 & 0.263 & 0.134 & 0.190 & 0.003 & 20 & NO & NO \\
\hline $\mathrm{NH}_{4}^{+}$Total & 0.101 & 0.022 & 0.007 & 0.029 & 0.111 & 0.155 & 0.055 & 21 & SÍ & SÍ \\
\hline OD & 0.000 & 0.000 & 0.000 & 0.000 & 0.000 & 0.000 & 0.007 & 24 & SÍ & SÍ \\
\hline Clorofila a & 0.001 & 0.000 & 0.000 & 0.005 & 0.002 & 0.285 & 0.006 & $24-32$ & SÍ & SÍ \\
\hline COT & 0.114 & 0.091 & 0.001 & 0.094 & 0.011 & 0.204 & 0.187 & 18 & SÍ & $\mathrm{NO}$ \\
\hline
\end{tabular}

G.V., Gradiente Vertical; G.H. Gradiente Horizontal

Tabla 10. Comparación entre puntos internos y entre salida LC - Pruebas t- pareada

\begin{tabular}{ccccccccccc}
\hline & $1.1 / 1.2$ & $1.1 / 2.1$ & $1.2 / 2.1$ & $1.2 / 2.2$ & $2.1 / 2.2$ & $2.1 /$ Sal & $2.2 /$ Sal & n & G.V. & G.H. \\
\hline DQO Total & 0.406 & 0.196 & 0.211 & 0.480 & 0.244 & 0.121 & 0.325 & $23-24$ & NO & NO \\
SST Total & 0.153 & 0.199 & 0.374 & 0.261 & 0.138 & 0.084 & 0.453 & $21-27$ & NO & NO \\
NO ${ }^{-}$ & 0.322 & 0.364 & 0.262 & 0.339 & 0.38 & 0.236 & 0.434 & 18 & NO & NO \\
NTK Total $^{N_{4}^{+} \text {Total }}$ & 0.396 & 0.074 & 0.215 & 0.293 & 0.558 & 0.005 & 0.054 & 20 & NO & NO \\
OD & 0.304 & 0.049 & 0.029 & 0.095 & 0.010 & 0.01 & 0.235 & 21 & SÍ & SÍ \\
Clorofila a & 0.000 & 0.036 & 0.000 & 0.115 & 0.000 & 0.000 & 0.237 & 24 & SÍ & SÍ \\
COT & 0.161 & 0.391 & 0.077 & 0.395 & 0.076 & 0.077 & 0.267 & $24-32$ & NO & NO \\
SOÍ & SÍ \\
\hline
\end{tabular}

G.V., Gradiente Vertical; G.H. Gradiente Horizontal

Tabla 11. Datos de entrada, verificación de modelos de carbono y nitrógeno

\begin{tabular}{|c|c|c|c|c|c|c|c|}
\hline $\mathrm{CO}_{2} \mathrm{COMOC}$ & COT & $\mathrm{NO}_{3} \mathrm{COMON}$ & $\mathrm{NH}_{4}{ }^{+} \mathrm{COMO} \mathrm{N}$ & NOD & NOP & \multicolumn{2}{|c|}{ CAUDALES } \\
\hline $\mathrm{gr} / \mathrm{m}^{3}$ & $\mathrm{gr} / \mathrm{m}^{3}$ & $\mathrm{gr} / \mathrm{m}^{3}$ & $\mathrm{gr} / \mathrm{m}^{3}$ & & $\mathrm{gr} / \mathrm{m}^{3}$ & $\mathrm{~m}^{3} /$ día & $\mathrm{m}^{3} /$ día \\
\hline 24.0 & 51.7 & 0.08 & 21.1 & 4.7 & 4.3 & 24.2 & 17.4 \\
\hline 26.4 & 60.6 & 0.08 & 22.8 & 4.1 & 3.8 & 24.6 & 18.1 \\
\hline 25.9 & 66.3 & 0.11 & 24.6 & 4.7 & 4.3 & 23.8 & 23.7 \\
\hline 24.0 & 60.2 & 0.08 & 18.4 & 3.0 & 2.7 & 23.7 & 26.2 \\
\hline 24.7 & 60.8 & 0.08 & 20.6 & 5.6 & 5.1 & 24.4 & 17.8 \\
\hline 22.8 & 48.5 & 0.09 & 18.4 & 5.9 & 5.4 & 21.9 & 24.8 \\
\hline 21.6 & 43.3 & 0.07 & 14.9 & 4.1 & 3.8 & 21.2 & 23.6 \\
\hline 25.9 & 68.43 & 0.62 & 28.10 & 2.4 & 2.2 & 30.2 & 22.5 \\
\hline 21.6 & 63.14 & 0.90 & 28.54 & 6.2 & 5.6 & 23.8 & 22.5 \\
\hline 26.9 & 63.07 & 0.75 & 18.9 & 4.7 & 4.3 & 25.5 & 21.9 \\
\hline 104.7 & 58.47 & 3.00 & 25.7 & 8.0 & 7.3 & 22.7 & 24.8 \\
\hline 96.8 & 53.80 & 0.70 & 26.2 & 7.7 & 7.0 & 27.3 & 25.8 \\
\hline
\end{tabular}

Tabla 12. Resultados de verificación

\begin{tabular}{|c|c|c|c|c|c|c|c|c|c|c|}
\hline \multirow[t]{3}{*}{$\mathrm{n}$} & \multicolumn{2}{|c|}{$\mathrm{CO}_{2}-\mathrm{C}$} & \multicolumn{2}{|c|}{ COT } & \multicolumn{2}{|c|}{$\mathrm{N}-\mathrm{NO}_{3}^{-}$} & \multicolumn{2}{|c|}{ NTK } & \multicolumn{2}{|c|}{$\mathrm{N}-\mathrm{NH}_{4}^{+}$} \\
\hline & \multicolumn{2}{|c|}{$\mathrm{gr} / \mathrm{m}^{3}$} & \multicolumn{2}{|c|}{$\mathrm{gr} / \mathrm{m}^{3}$} & \multicolumn{2}{|c|}{$\mathrm{gr} / \mathrm{m}^{3}$} & \multicolumn{2}{|c|}{$\mathrm{gr} / \mathrm{m}^{3}$} & \multicolumn{2}{|c|}{$\mathrm{gr} / \mathrm{m}^{3}$} \\
\hline & 1 & 2 & 1 & 2 & 1 & 2 & 1 & 2 & 1 & 2 \\
\hline 1 & 16.80 & 11.8 & 38.34 & 45.2 & 0.10 & 0.00 & 30.48 & 25.0 & 14.93 & 0.2 \\
\hline 2 & 18.24 & 13.8 & 39.36 & 52.5 & 0.09 & 0.00 & 31.61 & 25.7 & 13.61 & 0.3 \\
\hline 3 & 19.20 & 11.0 & 43.05 & 46.0 & 0.14 & 0.00 & 23.71 & 22.2 & 11.42 & 0.4 \\
\hline 4 & 19.20 & 9.0 & 44.78 & 38.2 & 0.12 & 0.00 & 21.45 & 14.7 & 13.17 & 0.5 \\
\hline 5 & 18.00 & 12.5 & 34.35 & 52.7 & 0.18 & 0.00 & 31.61 & 26.1 & 17.12 & 0.2 \\
\hline 6 & 19.44 & 7.9 & 30.73 & 29.7 & 0.14 & 0.00 & 22.58 & 17.9 & 13.61 & 0.4 \\
\hline 7 & 18.00 & 7.2 & 32.74 & 26.3 & 0.08 & 0.00 & 24.84 & 13.7 & 14.05 & 0.2 \\
\hline 8 & 23.76 & 15.7 & 52.79 & 63.1 & 0.24 & 0.01 & 37.26 & 28.0 & 23.71 & 0.5 \\
\hline 9 & 22.03 & 8.8 & 49.23 & 44.7 & 0.44 & 0.01 & 39.51 & 28.2 & 23.71 & 0.5 \\
\hline 10 & 23.04 & 13.3 & 54.67 & 50.0 & 0.16 & 0.01 & 52.36 & 21.5 & 15.92 & 0.2 \\
\hline 11 & 24.96 & 10.8 & 43.99 & 37.1 & 0.26 & 0.01 & 49.50 & 22.6 & 14.81 & 0.3 \\
\hline 12 & 24.48 & 12.2 & 57.24 & 40.0 & 0.12 & 0.00 & 47.60 & 25.8 & 15.55 & 0.3 \\
\hline Promedio & 20.60 & 11.16 & 43.44 & 43.78 & 0.17 & 0.003 & 34.38 & 22.60 & 20.53 & 0.32 \\
\hline Error & \multicolumn{2}{|c|}{$44 \%$} & \multicolumn{2}{|c|}{$20 \%$} & \multicolumn{2}{|c|}{$>50 \%$} & \multicolumn{2}{|c|}{$32 \%$} & \multicolumn{2}{|c|}{$>50 \%$} \\
\hline
\end{tabular}


DOI: https://doi.org/10.1016/S1405-7743(14)70353-1

Tabla 13. Prueba t-pareada. Medición - Simulación

\begin{tabular}{ccc}
\hline \multicolumn{2}{r}{ Variables comparadas* } & Sig. (2 colas) \\
\hline Par 1 & $\mathrm{CO}_{2}-\mathrm{CO}_{2} \mathrm{Sim}$ & .000 \\
Par 2 & $\mathrm{COT}-\mathrm{COTSim}$ & .938 \\
Par 4 & $\mathrm{NTK}-\mathrm{NTKSim}$ & .0019 \\
Par 5 & $\mathrm{NH}_{4}^{+}-\mathrm{NH}_{4}^{+} \mathrm{Sim}$ & .000 \\
\hline
\end{tabular}

* Para la variable $\mathrm{NO}_{3}{ }^{-}$no se cumplió hipótesis de normalidad, se corrió una prueba Wilcoxon y se logró que los valores medidos y los valores simulados fueran distintos.

Análisis de sensibilidad, AS

Las variables y parámetros en este análisis se presentan en las tablas 14 y 5, respectivamente; para su selección se analizaron los valores medidos en campo y se revisaron los parámetros que fueron objeto de calibración en otros estudios (Beran y Kargi, 2005; Sah, 2009; Senzia et al., 2002).

Tabla 14. Variables para AS

Variable

Oxígeno mínimo en LC

Temperatura

$\mathrm{pH}$

Radiación solar
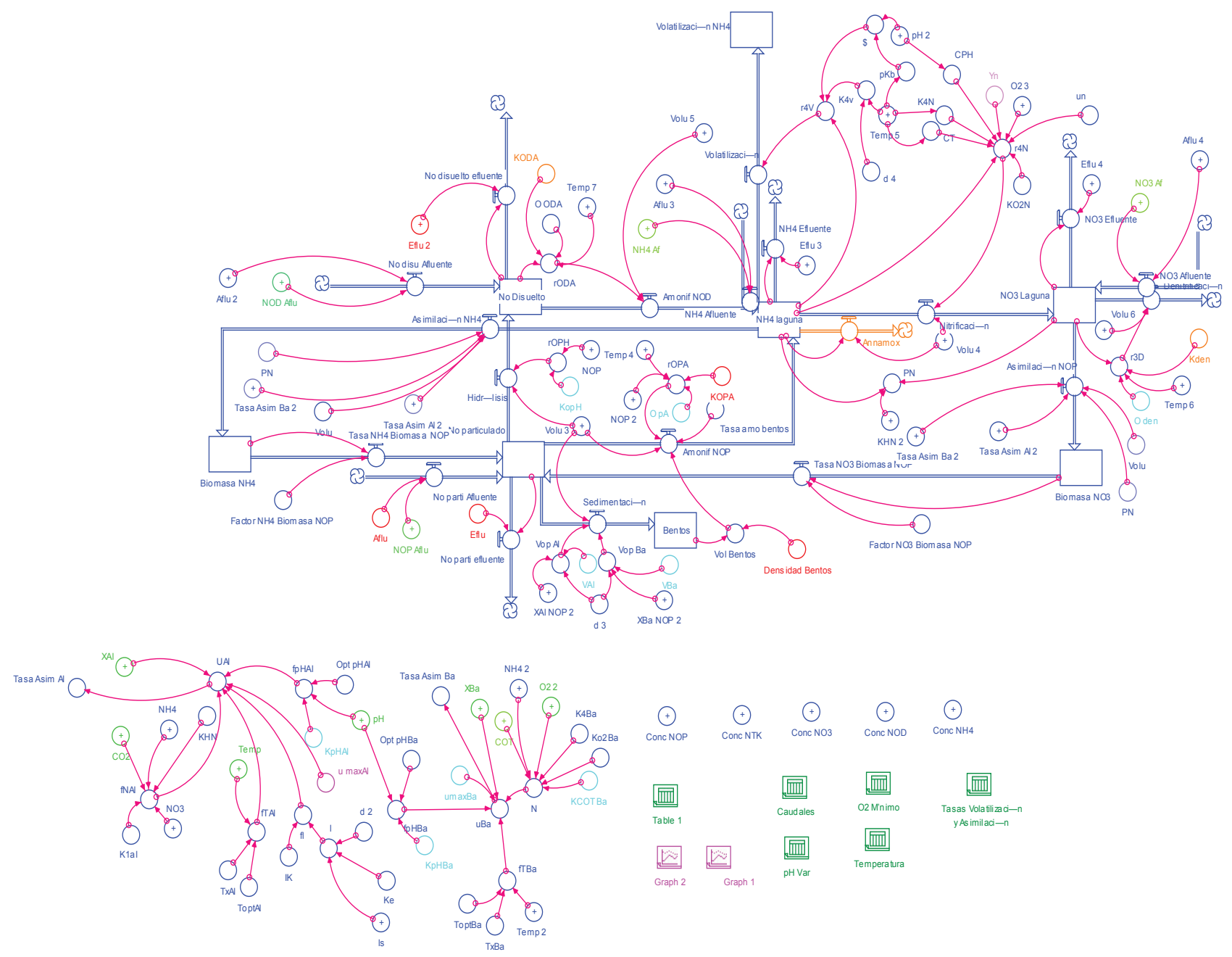

Figura 6. Modelo de nitrógeno ajustado

Las formas de biomasa $\mathrm{NH}_{4}^{+}$y $\mathrm{NO}_{3}^{-}$y sus tasas de conversión a NOP señaladas con línea punteada fueron las modificaciones planteadas al modelo inicial presentado antes. 
Tabla 15. Parámetros para AS

\begin{tabular}{ll}
\hline Parámetro & Definición \\
\hline $\mathrm{K}_{\text {COT Ba }}$ & Constante saturación media para la oxidación $(\mathrm{COT})$ por la bacterias. $(200,40-50)$ \\
$\mathrm{u}_{\max \text { Ba }}$ & Tasa crecimiento máximo para bacterias $(3.8,2-10)$ \\
$\mathrm{u}_{\text {max Al }}$ & Tasa crecimiento para microalgas $(0.5-2,2)$ \\
$\mathrm{K}_{\mathrm{CO} 2 \mathrm{Al}}$ & Constante saturación media CO2 tomado por microalgas $(1,0.5-0.6)$ \\
$\mathrm{K}_{1 \mathrm{OX}}$ & Tasa crecimiento $(0.1-1.2)$ \\
$\mathrm{V}_{\mathrm{Al}}$ & Velocidad sedimentación para el alga $(0.27-0.89)$ \\
$\mathrm{R}_{\mathrm{DBe}}$ & Tasa difusión del bentos $\mathrm{CO}_{2}(0.09)$ \\
$\mathrm{R}_{1 \mathrm{DCH} 4}$ & Tasa volatilización $\mathrm{CH}_{4}$ desde la columna de agua $(162.756)$ \\
$\mathrm{K}_{\mathrm{OPH}}$ & Tasa velocidad para la hidrólisis del NOP $(0.001-0.01)$ \\
$\mathrm{K}_{\mathrm{OPA}}$ & Tasa para la amonificación del NOP $(0.01-0.03)$ \\
$\mathrm{r}_{4 \mathrm{An}}$ & Tasa anammox $(0.000802)$ \\
$\mathrm{Y}_{\mathrm{bh}}$ & Relación sustrato / biomasa \\
\hline
\end{tabular}

Los modelos se corrieron empleando los valores promedio de los datos de entrada utilizados durante la etapa de verificación (tabla 16). Los resultados corresponden al promedio del último día de simulación (tabla 17). El oxígeno mínimo afectó los modelos hasta en $10 \%$ para COT y $\mathrm{NH}_{4}^{+}$, fue una relación inversa. El pH tuvo una relación inversa con $\mathrm{NO}_{3}^{-}$con diferencias superiores a $10 \%$. La temperatura afectó de manera inver- sa todas las especies analizadas. La temperatura afectó $\mathrm{NO}_{3}^{-}{ }^{-} \mathrm{NH}_{4}^{-}$en más de $10 \%$. La influencia de la radiación solar generó variaciones hasta de $25 \%$. Luego se efectuó el AS para los parámetros de interés y se relacionaron con base en las relaciones halladas entre ellos y las especies, así como lo encontrado en la etapa de verificación, se procedió a realizar la calibración de los modelos de C y N (tabla 18).

Tabla 16. Datos de entrada para AS

\begin{tabular}{ccccccccccc}
\hline & $\mathrm{CO}_{2} \mathrm{COMOC}$ & $\mathrm{COT}$ & $\mathrm{NO}_{3}{ }^{2}$ & $\mathrm{NTK}$ & $\mathrm{NH}_{4}^{+}$ & $\mathrm{NOT}$ & $\mathrm{NOD}$ & $\mathrm{NOP}$ & \multicolumn{2}{c}{ CAUDALES } \\
\hline & $\mathrm{gr} / \mathrm{m}^{3}$ & $\mathrm{gr} / \mathrm{m}^{3}$ & $\mathrm{gr} / \mathrm{m}^{3}$ & $\mathrm{gr} / \mathrm{m}^{3}$ & $\mathrm{gr} / \mathrm{m}^{3}$ & $\mathrm{gr} / \mathrm{m}^{3}$ & $\mathrm{gr} / \mathrm{m}^{3}$ & $\mathrm{gr} / \mathrm{m}^{3}$ & $\mathrm{gr} / \mathrm{m}^{3}$ & $\mathrm{gr} / \mathrm{m}^{3}$ \\
\hline Promedio & 24.90 & 58.19 & 1.36 & 37.26 & 27.51 & 9.74 & 5.10 & 4.64 & 24.44 & 22.42 \\
\hline
\end{tabular}

Tabla 17. Resultados AS - Variables

\begin{tabular}{|c|c|c|c|c|c|}
\hline & $\mathrm{CO}_{2}$ & COT & $\mathrm{NO}_{3}{ }^{=}$ & NTK & $\mathrm{NH}_{4}^{+}$ \\
\hline \multicolumn{6}{|c|}{ Caudal salida $\left(\mathrm{m}^{3} \mathrm{~d}^{-1}\right)$} \\
\hline 20.4 & 13.2 & 49.28 & 0.11 & 29.70 & 2.19 \\
\hline 21.4 & 12.0 & 46.28 & 0.07 & 28.18 & 1.10 \\
\hline 22.4 & 11.2 & 43.89 & 0.03 & 27.09 & 0.62 \\
\hline 23.4 & 10.6 & 41.57 & 0.01 & 26.16 & 0.36 \\
\hline 24.4 & 10.4 & 39.22 & 0.01 & 25.40 & 0.24 \\
\hline \multicolumn{6}{|c|}{ Oxígeno mínimo en $\mathrm{LC}\left(\mathrm{mg} \mathrm{L}^{-1}\right)$} \\
\hline 0.05 & 11.26 & 47.00 & 0.03 & 27.88 & 2.17 \\
\hline 0.638 & 11.19 & 44.81 & 0.03 & 27.31 & 1.11 \\
\hline 1.23 & 11.16 & 43.85 & 0.03 & 27.07 & 0.62 \\
\hline 1.81 & 11.15 & 43.29 & 0.03 & 26.96 & 0.34 \\
\hline 2.40 & 11.32 & 42.67 & 0.03 & 26.95 & 0.24 \\
\hline \multicolumn{6}{|c|}{ pH máximo (un) } \\
\hline 7.1 & 11.20 & 43.27 & 0.04 & 27.07 & 0.53 \\
\hline 8.1 & 11.20 & 43.28 & 0.03 & 27.07 & 0.53 \\
\hline 9.1 & 11.20 & 43.28 & 0.02 & 27.07 & 0.53 \\
\hline 10.1 & 11.20 & 43.28 & 0.01 & 27.06 & 0.53 \\
\hline 11.1 & 11.20 & 43.28 & 0.01 & 27.06 & 0.53 \\
\hline \multicolumn{6}{|c|}{ Temperatura máxima $\left({ }^{\circ} \mathrm{C}\right)$} \\
\hline 21 & 12.00 & 43.18 & 0.06 & 27.15 & 0.66 \\
\hline 22 & 11.50 & 43.31 & 0.03 & 27.07 & 0.53 \\
\hline 23 & 11.20 & 43.28 & 0.02 & 27.00 & 0.43 \\
\hline 24 & 10.90 & 42.99 & 0.01 & 26.94 & 0.34 \\
\hline 25 & 10.90 & 42.51 & 0.01 & 26.89 & 0.27 \\
\hline \multicolumn{6}{|c|}{ Radiación solar $\left(\mathrm{W} \mathrm{m}^{-2}\right)$} \\
\hline 368 & 13.70 & 39.28 & 0.40 & 38.06 & 19.90 \\
\hline 568 & 12.38 & 41.37 & 0.38 & 33.22 & 13.15 \\
\hline 768 & 11.16 & 43.28 & 0.13 & 25.64 & 2.18 \\
\hline 968 & 10.05 & 45.02 & 0.03 & 24.98 & 1.25 \\
\hline 1168 & 9.06 & 46.57 & 0.02 & 24.94 & 1.19 \\
\hline
\end{tabular}


DOI: https://doi.org/10.1016/S1405-7743(14)70353-1

Tabla 18. Resultados AS-Parámetros

\begin{tabular}{|c|c|c|c|c|c|}
\hline \multirow{2}{*}{ Parámetros } & \multicolumn{5}{|c|}{ Especies } \\
\hline & $\mathrm{CO}_{2}$ & COT & $\mathrm{NO}_{3}{ }^{=}$ & NTK & $\mathrm{NH}_{4}^{+}$ \\
\hline & 0 & $D$ & 0 & $D$ & $D$ \\
\hline 40 & 11.14 & 46.51 & 0.30 & 41.06 & 24.01 \\
\hline 80 & 11.19 & 48.50 & 0.31 & 42.45 & 25.95 \\
\hline 120 & 11.23 & 49.60 & 0.31 & 43.01 & 26.73 \\
\hline 160 & 11.25 & 50.27 & 0.31 & 43.31 & 27.15 \\
\hline 200 & 11.26 & 50.74 & 0.31 & 43.50 & 27.41 \\
\hline $\mathrm{u}_{\max B a}$ & 0 & $I$ & $D$ & $I$ & $I$ \\
\hline${ }_{2}^{\max \text { ва }}$ & 11.25 & 50.34 & 0.31 & 43.45 & 27.34 \\
\hline 3.5 & 11.19 & 48.35 & 0.31 & 42.49 & 26.01 \\
\hline 5 & 11.14 & 46.51 & 0.30 & 41.06 & 24.01 \\
\hline 6.5 & 11.09 & 44.82 & 0.30 & 38.68 & 20.69 \\
\hline 8 & 11.05 & 43.24 & 0.28 & 34.11 & 14.28 \\
\hline $\mathbf{u}_{\max \mathrm{Al}}$ & $I$ & $D$ & 0 & $I$ & $I$ \\
\hline $0.7^{\mathrm{Al}}$ & 15.29 & 39.55 & 0.31 & 44.12 & 28.27 \\
\hline 1.7 & 13.28 & 42.89 & 0.31 & 42.95 & 26.64 \\
\hline 2.7 & 11.14 & 46.51 & 0.30 & 41.06 & 24.01 \\
\hline 3.7 & 8.84 & 50.40 & 0.29 & 37.50 & 19.05 \\
\hline 4.7 & 6.41 & 54.49 & 0.23 & 29.14 & 7.23 \\
\hline $\mathbf{K}_{\mathrm{CO} 2 \mathrm{Al}}-\mathbf{K} \mathbf{1}_{\mathrm{Al}}$ & 0 & 0 & 0 & 0 & 0 \\
\hline 0.50 & 11.07 & 43.64 & 0.30 & 41.02 & 23.96 \\
\hline 0.75 & 11.11 & 46.57 & 0.30 & 41.04 & 23.98 \\
\hline 1 & 11.14 & 46.51 & 0.30 & 41.06 & 24.01 \\
\hline 1.25 & 11.18 & 46.45 & 0.30 & 41.07 & 24.03 \\
\hline 1.5 & 11.21 & 46.39 & 0.30 & 41.09 & 24.06 \\
\hline $\mathbf{K}_{10 x}$ & $D$ & $I$ & & & \\
\hline 0.075 & 10.78 & 47.13 & & & \\
\hline 0.0875 & 10.91 & 46.89 & & & \\
\hline 0.1 & 11.14 & 46.51 & & & \\
\hline 0.113 & 11.39 & 46.10 & & & \\
\hline 0.125 & 11.64 & 45.67 & & & \\
\hline $\mathbf{V}_{\mathrm{Al}}$ & 0 & $I$ & 0 & $I$ & $I$ \\
\hline $0.2^{\mathrm{Al}}$ & 11.19 & 48.29 & 0.30 & 42.68 & 24.19 \\
\hline 0.4 & 11.14 & 46.51 & 0.30 & 41.06 & 24.01 \\
\hline 0.6 & 11.09 & 44.87 & 0.30 & 39.93 & 23.88 \\
\hline 0.8 & 11.05 & 43.32 & 0.30 & 39.10 & 23.79 \\
\hline 1 & 11.01 & 41.88 & 0.30 & 38.47 & 23.72 \\
\hline $\mathbf{R}_{\mathrm{DBe}}-\mathbf{R}_{\mathrm{CO} 2 \mathrm{Be}}$ & 0 & 0 & & & \\
\hline 0.05 & 11.14 & 46.51 & & & \\
\hline 0.07 & Ídem & Ídem & & & \\
\hline 0.09 & Ídem & Ídem & & & \\
\hline 0.11 & Ídem & Ídem & & & \\
\hline 0.13 & Ídem & Ídem & & & \\
\hline $\mathbf{R} \mathbf{D}_{\mathrm{CH} 4}$ & 0 & I & & & \\
\hline 50 & 11.25 & 50.24 & & & \\
\hline 100 & 11.19 & 48.42 & & & \\
\hline 162.756 & 11.14 & 46.60 & & & \\
\hline 200 & 11.09 & 44.78 & & & \\
\hline 225 & 11.04 & 42.96 & & & \\
\hline $\mathbf{Y B}_{\mathrm{h}}$ & 0 & $D$ & & & \\
\hline 0.38 & 10.64 & 28.78 & & & \\
\hline 0.58 & 10.96 & 40.21 & & & \\
\hline 0.78 & 11.14 & 46.51 & & & \\
\hline 0.98 & 11.26 & 50.52 & & & \\
\hline 1.08 & 11.36 & 53.29 & & & \\
\hline $\mathbf{K}_{\mathrm{OPH}}$ & & & 0 & $D$ & $D$ \\
\hline 0.0075 & & & 0.30 & 40.99 & 23.98 \\
\hline 0.0244 & & & 0.30 & 41.03 & 24.00 \\
\hline 0.0413 & & & 0.30 & 41.06 & 24.01 \\
\hline 0.0581 & & & 0.30 & 41.09 & 24.02 \\
\hline 0.075 & & & 0.30 & 41.11 & 24.04 \\
\hline $\mathrm{K}_{\mathrm{OPA}}$ & & & 0 & $D$ & $D$ \\
\hline 0.01 & & & 0.30 & 40.79 & 23.59 \\
\hline 0.02 & & & 0.30 & 40.97 & 23.84 \\
\hline 0.03 & & & 0.30 & 41.15 & 24.08 \\
\hline 0.04 & & & 0.30 & 41.31 & 24.31 \\
\hline 0.05 & & & 0.30 & 41.47 & 24.53 \\
\hline $\mathbf{r}_{4 \mathrm{An}}$ & & & 0 & 0 & 0 \\
\hline 0.000102 & & & 0.30 & 40.79 & 23.59 \\
\hline 0.000352 & & & 0.30 & 40.97 & 23.84 \\
\hline 0.000602 & & & 0.30 & 41.15 & 24.08 \\
\hline 0.000852 & & & 0.30 & 41.31 & 24.31 \\
\hline 0.001100 & & & 0.30 & 41.47 & 24.53 \\
\hline
\end{tabular}

0: efecto nulo, D: relación directa, I: relación inversa 
El modelo de $C$ resultó sensible a: $\mathrm{K}_{\mathrm{COT} \mathrm{Ba}}, \mathrm{u}_{\max \mathrm{Ba}^{\prime}} \mathrm{u}_{\max \mathrm{A}{ }^{\prime}}$ $\mathrm{K}_{1 \mathrm{OX}}, \mathrm{V}_{\mathrm{Al}}, \mathrm{R}_{\mathrm{CH} 4}, \mathrm{YB}_{\mathrm{h}}$. Sin embargo, solo $\mathrm{u}_{\max \text { Al }} \mathrm{y} \mathrm{K}_{1 \mathrm{Ox}}$ tuvieron influencia sobre el $\mathrm{CO}_{2}{ }^{=}$. El modelo de $\mathrm{N}$ resultó sensible a: $\mathrm{K}_{\mathrm{COT} \mathrm{Ba}}, \mathrm{u}_{\max \mathrm{Ba}^{\prime}} \mathrm{u}_{\max \mathrm{Al}^{\prime}}, \mathrm{V}_{\mathrm{Al}}, \mathrm{K}_{\mathrm{OPH}}, \mathrm{K}_{\mathrm{OPA}}, \mathrm{r}_{4 \mathrm{An}}$. En ambos modelos la radiación solar, afectó sensiblemente todas las especies en estudio.

\section{Calibración}

Para el caso del modelo de C se calibró el COT. Se aceptó un error promedio hasta de $10 \%$ entre los datos del modelo y los de campo. Se adoptó un valor constante de $\mathrm{CO}_{2}{ }^{=}$correspondiente al promedio de los datos de campo, dado que la influencia de esta variable sobre el COT no fue significativa, el caudal de salida se adoptó igual al de entrada (tabla 19).

\begin{tabular}{|c|c|c|}
\hline Parámetro & Modelo C & Modelo N \\
\hline $\mathrm{u}_{\max \mathrm{Ba}}$ & 4 & 5 \\
\hline $\mathrm{u}_{\max \mathrm{Al}}$ & 1.7 & 2.7 \\
\hline $\mathrm{K}_{1 \mathrm{Ox}}$ & 0.125 & \\
\hline $\mathrm{V}_{\mathrm{Al}}$ & 0.2 & 0.2 \\
\hline $\mathrm{R} 1 \mathrm{D}_{\mathrm{CH} 4}$ & 75 & \\
\hline $\mathrm{Y}_{\mathrm{Bh}}$ & 0.6 & \\
\hline Radiación & 668 & 768 \\
\hline $\mathrm{K}_{\mathrm{OPH}}$ & & 0.01 \\
\hline $\mathrm{K}_{\mathrm{OPA}}$ & & 0.01 \\
\hline $\mathrm{r}_{4 \mathrm{An}}$ & & 0.000102 \\
\hline $\mathrm{K}_{\text {СОт Ва }}$ & & 40 \\
\hline
\end{tabular}

Los resultados de la calibración se probaron con una prueba t-pareada, relacionando los datos de campo y los datos de la simulación (tabla 20).

Tabla 20. Prueba $t$ - pareada. Datos campo - Datos simulados. Calibración LC

\begin{tabular}{ccc}
\hline & & Sig. (2colas) \\
\hline Par 1 & $\mathrm{NH}_{4}$ Campo- $\mathrm{NH}_{4}$ Sim & .386 \\
Par 2 & COTCampo-COTSim & .772 \\
\hline
\end{tabular}

Los valores arrojados por los modelos para las especies $\mathrm{NH}_{4}^{+}$y COT no presentaron diferencias con los valores medidos en campo. De acuerdo con los datos de campo solo se encontraron diferencias en el caso de la especie $\mathrm{NH}_{4}^{+}$entre las unidades piloto evaluadas LC y LBM, entre LBM y LC no se evidenciaron diferencias, por lo anterior se procedió a calibrar los modelos de C y N en la LBM. Para el caso del modelo de C, para la LBM se modificaron los valores del modelo topológico, volumen y profundidad; adicionalmente; se detectó que para un valor de $\mu$ Máx ${ }_{\mathrm{Al}}$ igual a $1.1 \mathrm{~d}^{-1}$, los resultados de las pruebas estadísticas fueron similares (tabla 21). Es importante mencionar este elemento, ya que abre la posibilidad de reducir el tamaño de esta unidad piloto, pues una tasa de menor producción de microalgas corresponde a la menor liberación de materia orgánica del sistema. Para el caso del $\mathrm{NH}_{4}{ }^{+}$se ajustaron los parámetros así: $\mathrm{KOpH}$ : 0.005, $\mu$ Máx $_{\mathrm{Al}}: 1.7$ y $\mathrm{r}_{4 \mathrm{An}}$ : 0.0. Los errores entre lo medido y lo simulado no superaron $10 \%$.

Tabla 21. Prueba t-pareada. Datos campo - Datos simulados. Calibración LBM

\begin{tabular}{ccc}
\hline & & Sig. (2 colas) \\
\hline Par 1 & $\mathrm{NH}_{4}$ Campo $-\mathrm{NH}_{4} \mathrm{Sim}$ & .348 \\
Par 2 & COTCampo - COTSim & .784 \\
\hline
\end{tabular}

Al igual que para el caso de la LC los modelos arrojaron resultados satisfactorios para COT y $\mathrm{NH}_{4}^{+}$de la LBM. El AS demostró que existen variables que le dan características redundantes al modelo, la selección de una $\mathrm{u}$ otra variable o parámetro hace que el modelo pueda ser afectado de igual forma por distintas vías; las etapas de sensibilidad y calibración pudieron reducir las suposiciones que otras experiencias de modelado ecológico han tomado (Sah, 2009), haciendo que existan más elementos de juicio para evaluar el modelo propuesto. Es necesario llevar a cabo ajustes y validación del componente de bentos de los modelos para corroborar el papel que juega, pues funciona de manera independiente de la columna de agua (Roy et al., 2008). El desarrollo de todas las etapas de construcción del modelo fueron determinantes para darle validez al ejercicio (Legendre y Legendre, 2004). La sensibilidad del modelo a variables como radiación solar se evidencia por otros autores (Sweeney et al., 2005) y en esta experiencia fue posible validarlo.

\section{Conclusiones}

La información que se obtuvo durante la investigación permitió seguir un proceso riguroso de verificación, validación y calibración de dos modelos topológicos de LFS, LC y LBM. Se encontraron diferencias significativas en la transformación de las sustancias de interés SST y $\mathrm{NH}_{4}^{+}$, entre los dos modelos topológicos. Los modelos ecológicos arrojaron resultados satisfactorios en la simulación de las variables de interés y lo medido en campo y contrastado con otras propuestas de modelado de LFS; su implementación sobre una plataforma hidrodinámica permitiría contar con una herramienta de diseño significativa en la perspectiva de proponer innovaciones de la tecnología. El modelado ecológico 
para LBM promete la posibilidad de reducir el tamaño de LFS promoviendo eliminación de $\mathrm{C}$ y $\mathrm{N}$ a distintos niveles utilizando facilidades como bafles y mallas. Es conveniente llevar a cabo el modelado bajo propuestas de comportamiento hidráulico distintas al de RCM. El modelado permite explorar las variables y parámetros que dan redundancia a estos sistemas y facilitan de esa manera su resiliencia.

\section{Agradecimientos}

El autor agradece al Departamento Administrativo de Ciencia, Tecnología e Innovación, Colciencias, Colombia, a ACUAVALLE S.A. ESP y a la Universidad del Valle por el soporte financiero y logístico de la investigación.

\section{APÉNDICE 1}

\section{Expresiones matemáticas de los modelos ecológicos para las formas de Carbono y Nitrógeno}

\section{Carbono orgánico}

$\mathrm{COT}=$ Afluente - efluente - respiración anaeróbica - respiración aeróbica (oxidación de materia orgánica) - consumo carbono asimilación bacterias + fotosíntesis (microalgas) + asimilación aeróbica + asimilación anaeróbica + transferencia de $\mathrm{CH}_{4}$ desde el bentos - sedimentación de materia orgánica al bentos - transferencia de $\mathrm{CH}_{4}$ desde la columna

\begin{tabular}{|c|c|c|c|c|}
\hline & Definición & Expresión & Expresión matemática extendida & Fuente \\
\hline$C_{\mathrm{Af}}$ & $\begin{array}{l}\text { Carbono orgánico total } \\
\text { afluente }\end{array}$ & $Q_{A f}{ }^{*} C O T_{\mathrm{Af}}$ & Dato de campo & \\
\hline$C_{\mathrm{Ef}}$ & $\begin{array}{l}\text { Carbono orgánico total } \\
\text { efluente }\end{array}$ & $Q_{E f}^{*} \mathrm{COT}_{\mathrm{Ef}}$ & Dato de campo & \\
\hline$R_{1 \text { Ran }}$ & $\begin{array}{l}\text { Tasa consumo de carbono } \\
\text { orgánico por anaerobiosis }\end{array}$ & $\mathrm{V} \times \mathrm{r}_{1 \mathrm{Ran}}$ & $r_{1 R a n}=C T^{*} X_{\text {co2 }}-r_{1 R a e}$ & (Picot et al., 2003) \\
\hline$R_{1 \text { Rae }}$ & $\begin{array}{l}\text { Tasa consumo de carbono } \\
\text { orgánico aerobiosis }\end{array}$ & $\mathrm{V} \times \mathrm{r}_{1 \text { Rae }}$ & $r_{1 R a e}=\left[K_{10 x}^{*} \theta_{1 O x}^{(T-20) *} \frac{O_{2}}{K_{1 O_{2}}+O_{2}}\right] * C O D$ & $\begin{array}{c}\text { (Mashauri y } \\
\text { Kayombo, 2002) }\end{array}$ \\
\hline$R_{1 R a s}$ & $\begin{array}{l}\text { Tasa consumo carbono } \\
\text { orgánico asimilación }\end{array}$ & $\mathrm{V} \times \mathrm{r}_{1}$ & $\begin{array}{c}r_{1}=\frac{X_{B a}}{Y_{\text {Bha }}} * \mu_{\text {maxBa }} * f\left(N_{B a}\right) f\left(T_{B a}\right) * f\left(p H_{B a}\right) \\
f\left(N_{B a}\right)=\left(\frac{N H 4}{K_{4 b a}+N H 4} * \frac{O_{2}}{k_{O 2 B a}+O_{2}} * \frac{C O T}{k_{C O T B a}+C O T}\right) f\left(T_{B a}\right) \\
e^{-2.3\left(\frac{T-T o p t B a}{T x B a-T o p t B a}\right)^{2}}, f\left(p H_{B a}\right)=\frac{K_{p H B a}}{K_{p H} B a+10^{(O p t p H B a-p H)}-1}\end{array}$ & $\begin{array}{l}\text { (Mashauri y } \\
\text { Kayombo, 2002; } \\
\text { Beran y Kargi, } \\
\text { 2005) }\end{array}$ \\
\hline$R_{1 C A}$ & $\begin{array}{l}\text { Tasa producción de carbono } \\
\text { orgánico en microalgas }\end{array}$ & $V \times r_{1 C A}$ & $\begin{array}{c}r_{1 C A}=\mu_{B A l} \mu_{A l}=X_{A l} * \mu_{\operatorname{maxAL}} * f\left(N_{A l}\right) * f(I) * f\left(T_{A l}\right) * f\left(p H_{A l}\right) \\
f\left(N_{A l}\right)=\frac{N H 4+N O_{3}}{K_{N A l}+N H 4+N O_{3}} * \frac{C O 2}{K_{C O 2 A l}+C O 2}, f(I)=\frac{I}{I_{K}} * e^{\left(1-\frac{I}{I_{K}}\right)}, I=I_{s}^{*} e^{-\left(k_{c}^{*} D\right)} \\
f(T)=e^{-2.3\left(\frac{T-T o p t A l}{T_{x A l}-T o p t A l}\right)^{2}}, f(p H)=\frac{K p H A l}{K p H A l+10^{\text {OptpHAl-pH })}-1}\end{array}$ & $\begin{array}{l}\text { (Jorgensen y } \\
\text { Bendoricchio, } \\
\text { 2001) }\end{array}$ \\
\hline$R_{1 C B}$ & $\begin{array}{l}\text { Tasa producción de carbono } \\
\text { orgánico por aerobiosis }\end{array}$ & $V \times r_{1 C B}$ & $\begin{array}{c}r_{1 C B}=\mu_{B}, \mu_{B}=X_{B a} \times \mu_{\operatorname{maxBa}} \times f\left(N O 3_{B a}\right) \times f\left(T_{B a}\right) \times f\left(O_{B a}\right) \times f\left(N H 4_{B a}\right), \\
f\left(N O 3_{B a}\right)=\left(\frac{N H 4}{K_{4 b a}+N H 4} * \frac{O_{2}}{k_{O 2 B a}+O_{2}} * \frac{C O T}{k_{C O T B a}+C O T}\right), f\left(T_{B a}\right)=e^{-2.3\left(\frac{T-T o p t B a}{T_{x B B}-T o p t B a}\right)^{2}} \\
f\left(p H_{B a}\right)=\frac{K_{p H B a}}{K_{p H} B a+10^{(O p t p H B a-p H)}-1}\end{array}$ & $\begin{array}{l}\text { (Beran y Kargi, } \\
\text { 2005) }\end{array}$ \\
\hline$R_{B B e}$ & $\begin{array}{l}\text { Tasa producción de carbono } \\
\text { orgánico por anaerobiosis }\end{array}$ & $V_{b} \times r_{B B e}$ & $V_{b}=$ Densidad Bentos ${ }^{*}$ Bentos, $r_{B B e}$ & Calibración \\
\hline$R D_{\text {CHABe }}$ & $\begin{array}{c}\text { Tasa transferencia de metano } \\
\text { desde el bentos }\end{array}$ & $V_{b} \times r_{C H 4 B e}$ & $V_{b}=$ Densidad Bentos $*$ Bentos, $r_{\text {CH4Be }}$ & Calibración \\
\hline$R V_{1 S}$ & $\begin{array}{l}\text { Tasa sedimentación de } \\
\text { biomasa }\end{array}$ & $V_{1 A l}+V_{1 B a}$ & 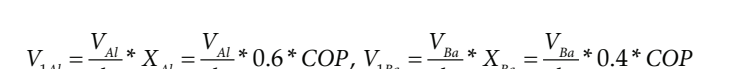 & (Senzia et al., 2002) \\
\hline$R D_{1 C H 4}$ & Tasa volatilización metano & & ${ }^{h} \quad$ Dato de campo ${ }^{h}$ & (Lasso, 2010) \\
\hline
\end{tabular}




\section{Carbono inorgánico}

C Inorgánico $=$ afluente - efluente + respiración aeróbica (oxidación de materia orgánica) + respiración anaeróbica fotosíntesis (asimilación microalgas) + transferencia de $\mathrm{CO}_{2}$ desde el bentos - transferencia de $\mathrm{CO}_{2}$ desde la columna + transferencia de $\mathrm{CO}_{2}$ desde la atmósfera - disolución de $\mathrm{CO}_{2}$ a carbonatos

$$
\frac{\partial C_{\text {Inor }}}{d t}=C I_{A f}-C I_{E f}+R_{1 \text { Rae }}+R_{1 \text { Ran }}-R_{F o}+R_{D B e}-R_{D L-A}+R_{D A-L}-R_{A l k}
$$

\begin{tabular}{|c|c|c|c|c|}
\hline & Definición & $\begin{array}{l}\text { Expresión } \\
\text { resumida }\end{array}$ & Expresión matemática extendida & Fuente \\
\hline$C I_{A f}$ & $\begin{array}{l}\text { Carbono inorgánico } \\
\text { total afluente }\end{array}$ & $Q_{A f} * \mathrm{CO}_{2 A f}$ & Dato de campo & \\
\hline$C I_{E f}$ & $\begin{array}{l}\text { Carbono inorgánico } \\
\text { total efluente }\end{array}$ & $Q_{A f}^{*} C O_{2 E f}$ & Dato de campo & \\
\hline$R_{1 \text { Ran }}$ & Respiración anaerobia & $V \times \mathrm{r}_{1 \operatorname{Ran}}$ & $r_{1 \text { Ran }}=\mathrm{COT}^{*} \mathrm{X}_{c o 2}-r_{1 R a e}$ & (Picot et al., 2003) \\
\hline$R_{1 \text { Rae }}$ & Respiración aerobia & $V \times \mathrm{r}_{1 \mathrm{Rae}}$ & $r_{1 \text { Rae }}=\left[K_{10 x} * \theta_{1 O x}^{(T-20)} * \frac{O_{2}}{K_{1 O_{2}}+O_{2}}\right] * C O D$ & $\begin{array}{c}\text { (Mashauri y } \\
\text { Kayombo, 2002) }\end{array}$ \\
\hline$R_{\mathrm{FO}}$ & Fotosíntesis & $V \times \mathrm{r}_{2 \mathrm{~F}}$ & $r_{2 F}=\mu_{A l}-X_{A l}^{*} K_{a l}$ & \\
\hline$R_{D B e}$ & $\begin{array}{l}\text { Liberación desde el } \\
\text { bentos } \mathrm{CO}_{2}\end{array}$ & $V_{b} \times \mathrm{r}_{\mathrm{CO} 2 \mathrm{Be}}$ & $V_{b}=$ Densidad Bentos ${ }^{*}$ Bentos, $r_{\mathrm{CO} 2 \mathrm{Be}}$ & Calibración \\
\hline$R_{D A-L}$ & $\begin{array}{l}\text { Transferencia } \\
\text { atmósfera - columna } \\
\text { de agua } \mathrm{CO}_{2}\end{array}$ & $V \times \mathrm{r}_{D A-L}$ & $\begin{array}{c}r_{2 D A-L}=\frac{12}{44} \frac{A}{V} K_{1 D}\left(C O_{2 s}-C O_{2 d}\right), K_{1 D}=\frac{44}{32} K_{o 2} \\
K_{O 2}=\left(0.728 W_{A-L}^{0.5}-0.31 W_{A-L}+0.0372 W_{A-L}^{2}\right)\left(1.0241^{(T-20)}\right) \\
C O_{2 S}=0.9664-0.0208 \mathrm{~T}\end{array}$ & (Beran y Kargi, 2005) \\
\hline$R_{D L-A}$ & $\begin{array}{c}\text { Transferencia columna } \\
\text { de agua - atmósfera } \\
\qquad \mathrm{CO}_{2}\end{array}$ & $V \times \mathrm{r}_{D L-A}$ & Ídem & Ídem \\
\hline$R_{A l k}$ & $\begin{array}{c}\text { Transformación } \mathrm{CO}_{2} \mathrm{a} \\
\text { carbonatos }\end{array}$ & $V \times \mathrm{r}_{2 \mathrm{Alk}}$ & $r_{2 A l k}=\mathrm{CO}_{2} * \mathrm{X}_{A l k}$ & (Picot et al., 2003) \\
\hline
\end{tabular}

\section{Nitrógeno amoniacal}

$\mathrm{NH}_{4}{ }^{+}=$Afluente - efluente + amonificación $\mathrm{NO}$ - nitrificación - asimilación biomasa - annamox - volatilización $\left.\frac{\partial \mathbf{N}_{4}}{\mathbf{d t}}=\mathbf{N H}_{4+\mathrm{Af}}-\mathbf{N H}_{4+\mathrm{Ef}}+\mathbf{R}_{\mathrm{A}}-\mathbf{R}_{4 \mathrm{C}}-\mathbf{R}_{4 \mathrm{~N}}-\mathbf{R}_{4 \mathrm{An}}-\mathbf{R}_{4 \mathrm{~V}}\right)$

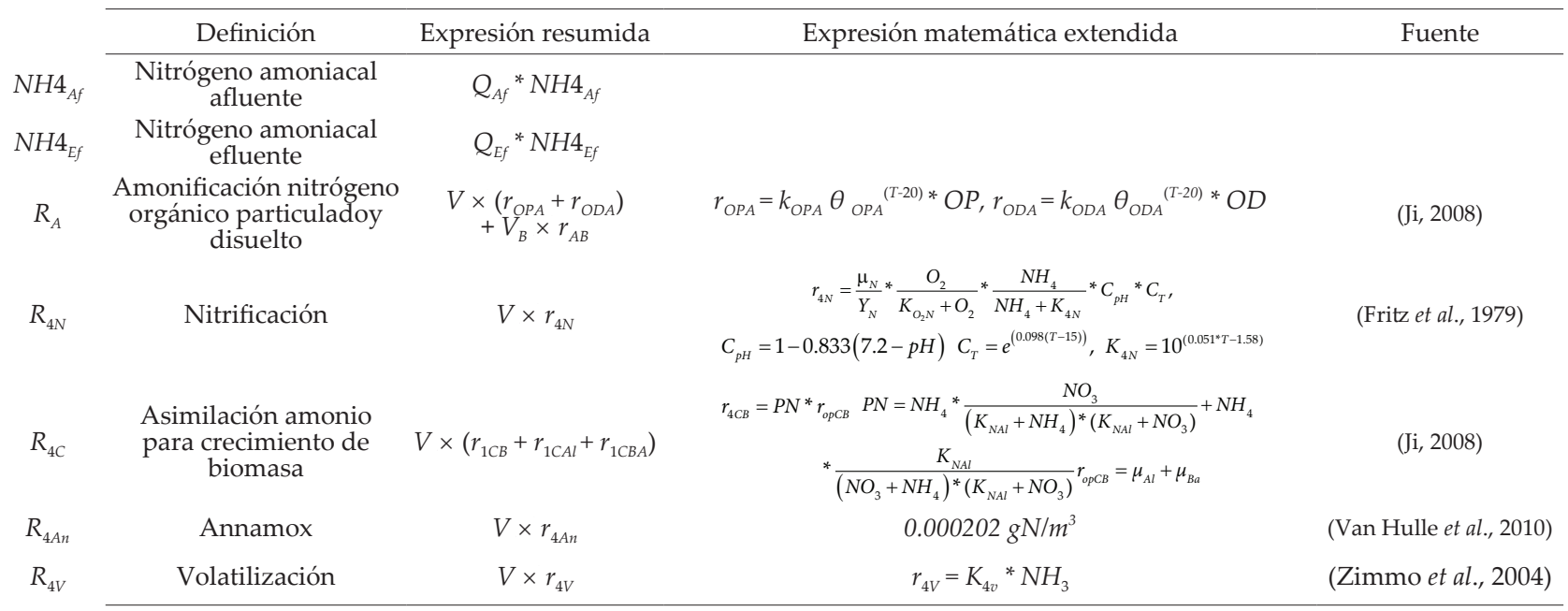




\section{Nitrógeno de nitratos}

$\mathrm{NO}_{3}^{-}=$Afluente + nitrificación - efluente - asimilación biomasa - denitrificación

\begin{tabular}{|c|c|c|c|c|}
\hline & Definición & Expresión resumida & Expresión matemática extendida & Fuente \\
\hline$N O 3_{A f}$ & $\begin{array}{l}\text { Nitrógeno nitratos } \\
\text { afluente }\end{array}$ & $Q_{A f}{ }^{*} N O 3_{A f}$ & & \\
\hline$N O 34_{E f}$ & $\begin{array}{l}\text { Nitrógeno nitratos } \\
\text { efluente }\end{array}$ & $Q_{E f}^{*} N O 3_{E f}$ & & \\
\hline$R_{4 N}$ & Nitrificación & $V \times r_{4 N}$ & & \\
\hline$R_{3 D}$ & Denitrificación & $\left(V \times r_{3 \mathrm{D}}\right)$ & $r_{3 D}=\theta_{\text {Den }}{ }^{(T-20)} * K_{D e n} * N_{3}$ & (Fritz et al., 1979) \\
\hline$R_{3 C B}$ & $\begin{array}{c}\text { Asimilación nitrato } \\
\text { para crecimiento de } \\
\text { biomasa }\end{array}$ & $\left(V \times r_{3 C B}\right)$ & $r_{3 C B}=(1-P N) *\left(r_{1 A l}+r_{1 C B}\right)$ & (Senzia et al., 2002) \\
\hline
\end{tabular}

\section{Nitrógeno orgánico particulado}

$\mathrm{NOP}=$ Afluente + biomasa - efluente - hidrólisis - amonificación NOP - sedimentación

\begin{tabular}{|c|c|c|c|c|}
\hline & Definición & Expresión resumida & Expresión matemática extendida & Fuente \\
\hline$N O P_{A f}$ & $\begin{array}{l}\text { Nitrógeno } \\
\text { nitratos afluente }\end{array}$ & $Q_{A f}{ }^{*} N O P_{A f}$ & & \\
\hline$N O P_{E f}$ & $\begin{array}{l}\text { Nitrógeno } \\
\text { nitratos efluente }\end{array}$ & $Q_{E f}{ }^{*} N O P_{E f}$ & & \\
\hline$R_{O P C B}$ & $\begin{array}{l}\text { Tasa de } \\
\text { producción de } \\
\text { biomasa }\end{array}$ & $V \times\left(r_{1 A l}+r_{1 C B}+r_{1 C B A}\right)$ & & \\
\hline$R_{\mathrm{OPH}}$ & $\begin{array}{l}\text { Hidrólisis } \\
\text { nitrógeno } \\
\text { orgánico } \\
\text { particulado }\end{array}$ & $V \times r_{\mathrm{OPH}}$ & $r_{\mathrm{OPH}}=K_{\mathrm{OPH}}{ }^{*} \mathrm{NOP}$ & $\begin{array}{c}\text { (Qitao y } \\
\text { Youngchul, 2009) }\end{array}$ \\
\hline$R_{O P A}$ & $\begin{array}{l}\text { Amonificación } \\
\text { nitrógeno } \\
\text { orgánico } \\
\text { particulado }\end{array}$ & $V \times r_{O P A}+T_{A m o B e n}$ & $r_{O P A}=K_{O P A} \theta_{O P A}^{(T-20) * N O P}$ & $\begin{array}{l}\text { (Jorgensen y } \\
\text { Bendoricchio, } \\
\text { 2001) }\end{array}$ \\
\hline$R_{\text {OPS }}$ & $\begin{array}{l}\text { Sedimentación } \\
\text { nitrógeno } \\
\text { orgánico } \\
\text { particulado }\end{array}$ & $V_{O P S}=V_{O P A L}+V_{O P B a c}$ & 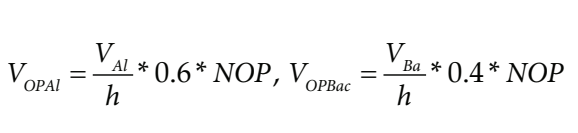 & (Senzia et al., 2002) \\
\hline
\end{tabular}




\section{Nitrógeno orgánico disuelto}

$$
\begin{aligned}
& \mathrm{NOD}=\text { Afluente }+ \text { hidrólisis }- \text { efluente }- \text { amonificación NOD } \\
& \frac{\partial N O D}{\partial t}=N O D_{A f}+R_{O P H}-N O D_{E f}-R_{O D A}
\end{aligned}
$$

\begin{tabular}{ccccc}
\hline & Definición & Expresión resumida & Expresión matemática extendida & Fuente \\
\hline$N O D_{A f}$ & $\begin{array}{c}\text { Nitrógeno orgánico } \\
\text { disuelto afluente } \\
\text { Nitrógeno orgánico } \\
\text { efluente }\end{array}$ & $Q_{A f}{ }^{*} N O D_{A f}$ & & \\
$N O D_{E f}$ & $Q_{E f}{ }^{*} N O D_{E f}$ & & \\
$R_{O P H}$ & $\begin{array}{c}\text { Hidrólisis nitrógeno } \\
\text { orgánico particulado }\end{array}$ & $V \times r_{O P H}$ & $r_{N O D}=k_{O D A} \theta_{O D A}{ }^{(T-20) *} N O D$ & $\begin{array}{c}\text { (Jorgensen y } \\
\text { Amonificación nitrógeno } \\
\text { orgánico disuelto }\end{array}$ \\
$R_{O D A}$ & $\mathrm{~V} \times r_{N O D}$ & Bendoricchio, 2001) \\
\hline
\end{tabular}

\section{Referencias}

Abbas H., Nasr R., Seif H. Study of Waste Stabilization Pond Geometry for the Wastewater Treatment Efficiency. Ecological Engineering, volmen 28, 2006: 25-34.

Alvarado A., Vedantam S., Goethals P. et al. A Compartmental Model to Describe Hydraulics in a Full-Scale Waste Stabilization Pond. Water Research, volumen 46, 2012: 521-530.

APHA-AWWA-WEF. Standart Methods for the Examination of Water and Wastewater, 21st ed., 2005.

Asaeda T., Van T. Modelling the Effects of Macrophytes on Algal Blooming in Eutrophic Shallow Lakes. Ecological Modelling, volumen 104, 1997: 264-287.

Banda C. Modern Design of Waste Stabilization Ponds in Warm Climates, Comparison with Traditional Design Methods, University of Leeds, Leeds, 2003.

Beran B., Kargi F. A Dynamic Mathematical Model for Wastewater Stabilization Ponds. Ecological Modelling, volumen 181, 2005: 39-57.

Bradley H.C., Nichols M.S. Nitrogen Content Bacterial Cells. Journal of Biological Chemistry, 1918.

Bravo A.M., Rodriguez P.H. Estudio del comportamiento hidrodinamico en lagunas facultativas secundarias para el tratamiento de aguas residuales domésticas, Unpublished Pregrado, Universidad del Valle, Cali, 2010.

Camargo M.A. Nitrogen Transformation Pathways and Removal Mechanisms in DomesticWastewater Treatment by Maturation Ponds, Unpublished Ph.D., University of Leeds, Leeds, 2008.

Chao X., Jia Y., Douglas F. et al. Numerical Modeling of Water Quality and Sediment Related Processes. Ecological Modelling, volumen 201, 2007: 385-397.

Chen C., Orlob G. Ecological Simulation of Aquatic Environments, Patten Systems Analysis in Ecology 3, 1975.

Fagerbakke K.M., Heldal M., Norland S. Content of Carbon, Nitrogen, Oxygen, Sulfur and Phosphorus in Native Aquatic and Cultured Bacteria. Aquatic Microbial Ecology, volumen 10, 1996:15-27.

Farrás L.E.P. Teoría de la sedimentación, Buenos Aires, Universidad Tecnológica Nacional, Facultad Regional Bahía Blanca, 2005.

Fichmann J.J. Estimación de los coeficientes cinéticos de transformación de nitrógeno en lagunas facultativas secundarias utilizadas en el tratamiento de aguas residuales municipales, Unpublished MSc, Universidad del Valle, Cali, 2012.

Fritz J.J., Middleton A.C., Meredith D.D. Dynamic Process Modelling of Wastewater Stabilization Ponds. Journal WPCF, volumen 51, 1979: 2724-2743.

Heaven S.B., C. Zotova E. Light Attenuation Parameters for Waste Stabilization Ponds. Water Science and Technology, volumen 41, 2005:143-152.

Henzen M., Gujer W., Mino T. et al. Activated Sludge Model, Núm. 2, 1995.

Houweling D., Kharoune L., Escalas A., et al. Dynamic Modelling of Nitrification in an Aerated Facultative Lagoon. Water Research, volumen 42, 2008: 424-432.

Isse-Systems: Stella Technical Documentation, en: edited by, 2005.

Jamu D.M., Piedrahita R.H. An Organic Matter and Nitrogen Dynamics Model for the Ecological Análisis of Integrated Aquaculture/Agriculture Systems: I Model Development and Calibration. Environmental Modelling \& Software, volumen 17, 2002: 571-582.

Janex-Habibi M.L., Huyard A., Esperanza M. et al. Reduction of Endocrine Disruptor Emissions in the Environment: The Benefit of Wastewater Treatment. Water Research, volumen 43, 2009:1565-1576.

Jenkins B.D., Zehr J.P. Molecular Approaches to the Nitrogen Cycle, in Nitrogen in the Marine Environment, 2008.

Ji Z.G. Hydrodynamics and Water Quality. Modeling Rivers, Lakes, and Estuaries,Wiley-Interscience, New Jersey, John Wiley and Sons, 2008.

Jorgensen S.E., Bendoricchio G. Fundamentals of Ecological Modelling, 3rd ed., Kidlington, UK, Elsevier, 2001.

Kayombo S., Mbwette T.S.A., Mayo A.W. et al. Modelling Diurnal Variation of Dissolved Oxygen in Waste Stabilization Ponds. Ecological Modelling, volumen 127, 2000: 21-31.

Kayombo S., Mbwette T., Katima J. et al. Effects of Substrate Concentrations on the Growth of Heterotrophic Bacteria and Algae in Secondary Facultative Ponds. Water Research, volumen 37, 2003: 2937-2943.

Lampert W., Sommer U. Limnoecology. The Ecology the Lakes and Streams, 2nd ed., New York, USA, Oxford University Press, 2007. 
Lasso A.P. Cámaras estáticas para la estimación de emisiones de gases de efecto invernadero en lagunas de estabilización para tratamiento de aguas residuales domésticas, (tesis), Universidad del Valle, Cali, 2010

Legendre P., Legendre L. Numerical Ecology: Developments in Environmental Modelling, 20, Amsterdam, The Netherlands, Elsevier, 2004

Mara D.D. Domestic Wastewater Treatment in Developing Countries, en: Earthscan/James y James, UK, 2004.

Mashauri D.A., Kayombo S. Application of the two Coupled Models for Water Quality Management: Facultative Pond Cum Constructed Wetland Models. Physics and Cchemistry of the Earth, volumen 27, 2002: 773-781.

Metcalf, Eddy, 1995.

Mišurcová L., Kráčmar S., Klejdus B. et al. Nitrogen Content, Dietary Fiber and Digestibility in Algal Food Products. Czech J. Food Sci., volumen 28, 2010: 27-35.

Muñoz R., Guieysse B. Algal-Bacterial Processes for the Treatment of Hazardous Contaminants: A Review, 17, 2006.

Pepperell C., Foley J., Choong L. et al. An Improved Method for Estimating Fugitive Methane Emissions from Wastewater Treatment Lagoons, en: 9th IWA Specialist Group Conference on Waste Stabilisation Ponds, Adelaida, Australia, 2011, pp. 339-336.

Pereira A., Duarte P., Norro A. Different Modelling Tools of Aquatic Ecosystems: a Proposal for a Unified Approach. Ecological Informatics, volumen 1, 2006: 407-421.

Picot B., Paing J., Sambuco J.P., et al. Biogas Production, Sludge Accumulation and Mass Balance of Carbon in Anaerobic Ponds. Water Science \& Technology, volumen 48, 2003: 243250.

Qitao Y.C., Youngchul H.K. Modeling Nitrogen Removal in Water Hyacinth Ponds Receiving Effluent from Waste Stabilization Ponds. Ecological Engineering, volumen 35, 2009: 75-84.

Reed S., Crites R., Middlebrooks E. Natural Systems for Waste Management And Treatment, McGraw-Hill Professional, 1998.

Rees W.E. Human Nature, Eco-Footprints and Environmental Injustice. Local Environment, volumen 13, 2008: 685-701.

Roy M., Mandal S., Ray S. Detrital Ontogenic Model Including Decomposer Diversity. Ecological Modelling, volumen 215, 2008: 200-206

Sah L. 3D Modelling of Secondary Facultative Ponds. (tesis), UNESCO-IHE, Delft, 2009.

Senzia M., Mayo A., Mbwette T. et al. Modelling Nitrogen Transformation and Removal in Primary Facultative Ponds. Ecological Engineering, volumen 154, 2002.

Shen L.D., He Z.F., Zhu Q. et al. Microbiology, Ecology, and Application of the Nitrite-Dependent Anaerobic Methane
Oxidation Process. Frontiers in Microbiology, volumen 3, 2012: 1-5.

Shilton A., Harrison J. Guidelines for the Hydraulic Design of Waste Stabilisation Ponds. Palmerston North, New Zealand, Institute of Technology and Engineering, 2003.

Sweeney D.G., Cromar N.J., Fallowfield H.J. et al. Profiling and Modelling of Thermal Changes in a Large Waste Stabilisation Pond. Water Science \& Technology, volumen 51, 2005:163-172.

Tchobanoglous G., Burton F.L., Metcalf et al. Wastewater Engineering: Treatment and Reuse, McGraw-Hill, 2004.

Tortora G., Funke B., Case C. Introducción a la microbiología, editorial médica panamericana, 2007.

Van Hulle S.W., H. Meesschaert B., Vanrolleghema P. et al. Engineering Aspects and Practical Application of Autotrophic Nitrogen Removal from Nitrogen Rich Streams. Chemical Engineering Journal, volumen 162, 2010: 1-20.

Von Sperling M. Design of Facultative Ponds Based on Uncertainly Analysis. Water Science Technology, volumen 33, 1996: 41-47.

Wallace S., Austin D. Emerging models for Nitrogen Removal in Treatment Wetlands. Journal of Environmental Health, volumen 71, 2008: 10-16.

Zima P., Makinia J., Swinarski M. et al. Combining Computational Fluid Dynamics with a Biokinetic Model for Predicting Ammonia and Phosphate Behavior in Aeration Tanks. Water Environmental Research, volumen 81, 2009: 2353-2362.

Zimmo O., Steen N., Gijzen H. Comparison of Ammonia Volatilisation Rates in Algae and Duckweed-Based Waste Stabilisation Ponds Treating Domestic Wastewater. Water Research, volumen 37, 2003: 4587-4594.

Zimmo O.R., Van Der Steen N.P., Gijzen H.B. Nitrogen Mass Balance Across Pilot-Scale Algae and Duckweed-Based Wastewater Stabilisation Ponds. Water Research, volumen 38, 2004: 913-920.

\section{Este artículo se cita:}

\section{Citación estilo Chicago}

Aponte-Reyes, Alexander. Desarrollo de modelos ecológicos para carbono y nitrogeno en lagunas facultativas secundarias. Ingeniería Investigación y Tecnología, XV, 03 (2014): 437-456.

\section{Citación estilo ISO 690}

Aponte-Reyes A. Desarrollo de modelos ecológicos para carbono y nitrogeno en lagunas facultativas secundarias. Ingeniería Investigación y Tecnología, volumen XV (número 3), julio-septiembre 2014: 437-456.

\section{Semblanza del autor}

Alexander Aponte-Reyes. Es ingeniero sanitario, con maestría en ingeniería sanitaria y ambiental, actualmente es candidato a doctor en la misma área. Sus áreas de trabajo son el abastecimiento de agua y el control de la contaminación ambiental a través de un enfoque ecológico, incluyendo proyectos de investigación y desarrollo sobre tecnologías naturales, el empoderamiento de la comunidad y el comportamiento higiénico. Su perfil profesional incluye proyectos de investigación y la experiencia en las actividades relacionadas con la promoción y ejecución de proyectos de desarrollo, orientados hacia las zonas rurales, municipios pequeños y medianos. 